\title{
Knowledge Management for Self-Organised Resource Allocation
}

\author{
DAVID BURTH KURKA, Imperial College London, UK \\ JEREMY PITT, Imperial College London, UK \\ JOSIAH OBER, Stanford University, USA
}

Many instances of socio-technical systems in the digital society and digital economy require some form of self-governance. Examples include community energy systems, peer production systems, participatory sensing applications, and shared management of communal living areas or workspace. Such systems have several features in common, of which three are that they are rule-oriented, self-organising and value-sensitive, and in operation, this combination of features entails self-modification of the rules in order to satisfice a changeable set of values. This presents a fundamental dilemma for systems design. On the one hand, the system must be sufficiently unrestricted (resilient, flexible) to enable a diverse group but with a shared set of congruent values to achieve their joint purposes in collective action situations. On the other hand, it must be sufficiently restricted (stable, robust) to prevent a subset of the group from exploiting self-determination 'against itself' and usurp control of the system for the benefit of their own narrow interests. To address this problem, we consider a study of classical Athenian democracy which investigates how the governance model of the city-state flourished. The work suggests that exceptional knowledge management, i.e. making information available for socially productive purposes, played a crucial role in sustaining its democracy for nearly 200 years, by creating processes for aggregation, alignment and codification of knowledge. We therefore examine the proposition that some properties can be generalised to resolve the rule-restriction dilemma by establishing a set of design principles intended to make knowledge management processes open, inclusive, transparent and effective in self-governed social technical systems. We operationalise three of these principles in the context of a collective action situation, namely self-organised common-pool resource allocation, and present the results of a series of experiments showing how knowledge management processes can be used to obtain robust solutions for the perception of fairness, allocation decision and punishment mechanisms. By applying this operationalisation of the design principles for knowledge management processes as a complement to institutional approaches to governance, we demonstrate empirically how it can satisfice shared values, distribute power fairly, and apply "common sense" in dealing with rule violations. We conclude by arguing that this approach to the design of socio-technical systems can provide a balance between restricted and unrestricted self-modification of conventional rules, and can thus provide the foundations for sustainable and democratic self-governance in socio-technical systems.

\section{CCS Concepts: • Computing methodologies $\rightarrow$ Multi-agent systems; $\bullet$ Social and professional topics} $\rightarrow$ Socio-technical systems; • Computer systems organization $\rightarrow$ Self-organizing autonomic computing

Additional Key Words and Phrases: Knowledge Management, Common-Pool Resource Allocation, NormGoverned Systems

Authors' addresses: David Burth Kurka, Imperial College London, Department of Electrical and Electronic Engineering, London, SW7 2BT, UK, d.kurka@imperial.ac.uk; Jeremy Pitt, Imperial College London, Department of Electrical and Electronic Engineering, London, SW7 2BT, UK, j.pitt@imperial.ac.uk; Josiah Ober, Stanford University, Department of Political Science, Stanford, CA, 94305, USA, jober@stanford.edu.

Permission to make digital or hard copies of all or part of this work for personal or classroom use is granted without fee provided that copies are not made or distributed for profit or commercial advantage and that copies bear this notice and the full citation on the first page. Copyrights for components of this work owned by others than ACM must be honored Abstracting with credit is permitted. To copy otherwise, or republish, to post on servers or to redistribute to lists, requires prior specific permission and/or a fee. Request permissions from permissions@acm.org.

(C) 2019 Association for Computing Machinery.

1556-4665/2019/5-ART1 \$15.00

https://doi.org/0000001.0000001

ACM Trans. Autonom. Adapt. Syst., Vol. 1, No. 1, Article 1. Publication date: May 2019 


\section{ACM Reference Format:}

David Burth Kurka, Jeremy Pitt, and Josiah Ober. 2019. Knowledge Management for Self-Organised Resource Allocation. ACM Trans. Autonom. Adapt. Syst. 1, 1, Article 1 (May 2019), 42 pages. https://doi.org/0000001. 0000001

\section{INTRODUCTION}

Many instances of socio-technical systems in the digital society and digital economy require some form of (potentially unrestricted) self-governance. Examples include community energy systems providing localised distributed and storage of energy [Steghöfer et al. 2013] which involve people, 'smart' meters and programmable appliances; peer production systems with local pooling of tools, machinery and resources [Rychwalska and Roszczynska-Kurasinska 2017] or knowledge commons such as Wikipedia [Hess and Ostrom 2007]; participatory sensing applications [Burke et al. 2006] where people pool raw sensor data in exchange for some beneficial service based on aggregated or processed data; and shared management of a communal living area or workspace [Picard 1997].

These systems have at least three features in common. Firstly, they are rule-oriented systems, and these rules are mutable and mutually agreed. Mutability implies that there are meta-rules for changing the rules (and meta-meta-rules, etc.), while mutual agreement implies that since these are conventional rather than physical rules, they can be violated or repudiated, so that there are also (multi-level) meta-rules for breaking the rules. Importantly, systems of meta-rules can be codified, for example using logical axioms [Artikis 2012] or grammars [Crawford and Ostrom 1995]. Secondly, these are self-organising systems: there is no centralised controller and, when it comes to the rules, their application, selection and modification is performed by the participants themselves. Thirdly, these are value-sensitive systems: an underpinning reason for having rules is so that the participants can satisfy (or at least satisfice) their shared values. These values may not even be explicitly referenced by the rules but values and rules should still be aligned.

These Self-Governing Socio-Technical Systems need to be regulated by a type of governance based on the codification of conventional rules, which are self-organised and enacted by the system's participants themselves, and should respect some (perhaps implicit) value, like fairness or sustainability. One approach to design and development of these systems, complementing for example value-sensitive design [Friedman et al. 2013], is to use Ostrom's design principles for self-governing institutions [Ostrom 1990].

Yet, attention should be given to some risks, including: the paradox of self-amendment [Suber 1990], where the enactment of a rule contains clauses for its own amendment, to the extent that the rules are logically contradictory, inconsistent or incomplete; path dependency, where a prior decision or series of decisions results in the transaction costs of change being greater than the benefits (to the current participants) of changing, so a supposedly mutable system stagnates [Collier and Collier 1991]; and the iron law of oligarchy [Michels 1915], which maintains that any conventional rule-based system, no matter how 'democratically' conceived and founded, will tend towards oligarchy as a subset of the participants manipulate the system in favour of their own sectional interests (i.e. prioritising or promoting values which are not shared by the collective as a whole).

Therefore, a fundamental dilemma in the design of such systems is presented, which necessarily require self-modification of a set of conventional rules. On the one hand, the system must be sufficiently unrestricted (resilient, flexible) to enable a diverse group of people but with a shared set of congruent values to achieve their joint purpose(s) in collective action situations. On the other hand, the system must be sufficiently restricted (stable, robust) to prevent a subset of the group from exploiting this openness 'against itself', as it were, by usurping control of the system and running it for the benefit of their own narrow (un-shared) interests. In addition, restrictions (and supervision) may also be necessary to avoid path dependency and the paradox of self-amendment, 
and to address issues of scale in nested enterprises and attention as a scarce resource [Lanham 2006].

The solution that is proposed in this paper is based on the operationalisation of Knowledge Management (KM) principles, derived from a study of classical Athenian democracy [Ober 2008]. Athenian democracy was a governance model for a city-state that was successful and sustained for over 180 years, in the face of competition from other Hellenic city-states and external powers. The analysis proposes that classical Athens' superior economic and military performance was, at least in part, a product of democratic institutions and civic culture and its "use of knowledge in society" [Hayek 1945].

The investigation, however, shows that the idea of "one citizen one vote" was not the primary reason for this success (although it contributed). Instead, it was openness, transparency and inclusivity across multiple inter-dependent knowledge management processes, i.e. making information available for socially productive purposes. The historical analysis show how Athenians achieved unprecedented information processing abilities, transforming raw data and unprocessed information into politically valuable knowledge, by the consolidation of methods that aggregated, aligned, and codified knowledge.

We examine the proposition that similar properties of knowledge management processes can be applied to socio-technical systems and be used to resolve the rule-restriction dilemma for selfgovernance, taking into account the necessary considerations implied in moving from an antique to a digital context.

Thus, considering the challenges and reality of digital societies, we consider a set of eight design principles for developing and protecting open, inclusive and transparent knowledge management processes for democratic and sustainable self-organising socio-technical systems [Pitt et al. 2017]. As a way of demonstrating their applicability, we operationalise three of the principles in the context of a collective action situation, namely common-pool resource allocation - a classic, but challenging problem of group coordination and self-organisation [Araral 2014], and describe the implementation of a multi-agent simulation using this operationalisation.

Table 1. Summary of the KM design principles being considered in this work, emphasising the information being considered as input and the socially productive purpose as outcome.

\begin{tabular}{cccc}
$\begin{array}{c}\text { KM } \\
\text { Design } \\
\text { Principle }\end{array}$ & Algorithm & $\begin{array}{c}\text { Information made } \\
\text { available }\end{array}$ & $\begin{array}{c}\text { Socially productive } \\
\text { purpose }\end{array}$ \\
\hline 6 & $\begin{array}{c}\text { Voices of Justice } \\
(\text { VoJ) }\end{array}$ & $\begin{array}{c}\text { Individual } \\
\text { perception of } \\
\text { fairness }\end{array}$ & $\begin{array}{c}\text { Feedback of system's } \\
\text { operation }\end{array}$ \\
7 & $\begin{array}{c}\text { Distributed } \\
\text { Distributive Justice } \\
\text { (DDJ) }\end{array}$ & $\begin{array}{c}\text { Legitimate claims of } \\
\text { justice }\end{array}$ & $\begin{array}{c}\text { Decision of resource } \\
\text { allocation policies }\end{array}$ \\
8 & $\begin{array}{c}\text { Flexible Monitoring } \\
\text { and Sanctioning } \\
\text { (FMS) }\end{array}$ & $\begin{array}{c}\text { Behaviour towards } \\
\text { norms (compliance } \\
\text { and non-compliance) }\end{array}$ & $\begin{array}{c}\text { Definition of criteria } \\
\text { for rule enforcement }\end{array}$ \\
& & &
\end{tabular}

For each KM design principle operationalised, we present an algorithm that considers different types of information present in the resource allocation problem, and transforms it into valuable knowledge so it can be used for a socially productive purpose - as summarised in Table 1 . The experiments show that such KM design principles operationalisations can be used to obtain robust 
solutions for the problems of (a) perception of fairness, in which agents connected in a social network have different perceptions and opinions of resource allocation policies; (b) allocation decision, in which agents collectively define rules for resource allocation and distribution; and (c) punishment mechanisms, in which non-compliance is managed by appropriate monitoring and sanctioning.

Thus, by applying this operationalisation as a complement to institutional approaches to governance [Ostrom 1990], we demonstrate empirically how it can satisfice shared values, distribute power fairly, and apply "common sense" in dealing with rule violations.

This paper is organised as follows. Section 2 brings the theoretical background used in the subsequent sections, by defining the knowledge processes theory, starting from the analysis of Classical Athens [Ober 2008], culminating in the specification of eight design principles for sociotechnical systems [Pitt et al. 2017]. To investigate applications of the proposed principles, a commonpool resource allocation game is introduced and formalised in Section 3 and used as base for the investigation of operationalisation of three of the eight principles presented in Section 2.2. Principle 6 (Voices of Justice), Principle 7 (Distributed Distributive Justice) and Principle 8 (Flexible Monitoring and Sanctioning) are investigated in Sections 4, 5 and 6 respectively. For each principle a motivating scenario is given, justifying the implementation of solutions based on the outlined principles. In each case, a solution strategy based on the corresponding principle is shown, with experimental results and discussion. Finally, Section 7 concludes this work with the argument that this approach to the design of socio-technical systems can provide the foundations for sustainable and democratic self-governance in socio-technical systems.

\section{BACKGROUND AND MOTIVATION}

\subsection{Knowledge Management in Classical Athens}

It has been shown that classical Athenian democracy (ca. 500-320 B.C.), on a number of independent metrics, massively outperformed its rival city states, economically, architecturally, militarily, and diplomatically; despite a relative parity in territorial size, population density, cultural development, and availability of mineral resources [Ober 2008]. The exceptional success of Athenian democracy was attributed to the greater social benefits derived from higher levels of cooperation. This in turn was based on the Athenians' superior capacity for resolving public collective action problems, which itself was a product of special features of their participatory and deliberation model of self-governance. One of the most important of these special features was the distinctive Athenian system for organising useful knowledge.

The analysis in [Ober 2008] shows that the Athenians developed highly effective, transparent and interdependent epistemic Knowledge Management processes. By Knowledge Management is meant the process of "making information available for socially productive purposes through individual choices made in the context of institutional processes, involving both innovation and learning" [Ober 2008, p. 18].

Ober shows that the integration of dispersed and latent technical knowledge with social knowledge and shared values was key to successful democratic decision-making. Thus, Athens revealed notable information processing ability, transforming raw data (i.e. facts about events) and unprocessed information (i.e. data that have been given relevance and purpose) into politically valuable knowledge (i.e. a matrix of new experiences and information [Brown and Duguid 2000; Davenport and Prusak 1998; Dixon 2000; Page 2007]).

That transformation was carried out through processes of: knowledge aggregation, by which dynamic knowledge, created by and between citizens, delivered the 'right' course of action that 'best' represented or served their shared values; knowledge alignment, which used mutual knowledge to 


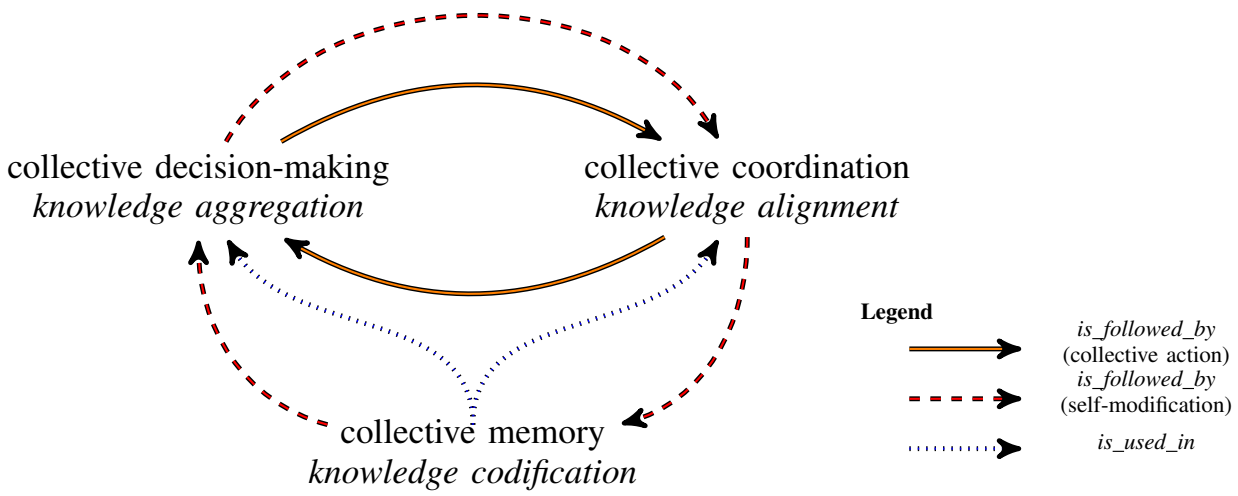

Fig. 1. Athenian knowledge management processes (adapted from [Ober 2008])

coordinate individuals in successfully satisfying that course of action; and knowledge codification, which standardised institutional structures and procedures, increasing openness and effectiveness and supporting inclusiveness, verification and accountability. The relationship between these processes is illustrated in Figure 1. Note the arrows represent sequencing rather than dataflows. There are two sequences: in the inner sequence (solid arrows), knowledge aggregation precedes knowledge alignment and both processes are supported by codified knowledge (dotted arrows) to solve collective action problems (e.g. the distribution of common-pool resources). However, codified knowledge itself can be modified by a "knowledge aggregation - knowledge alignment" sequence, as represented by the outer sequence (dashed arrows), used for selecting and modifying the rules for the distribution of common-pool resources.

2.1.1 Knowledge Aggregation (KAgg). Classical Athenian structures and processes for decisionmaking included effective social/knowledge networks based on civic sub-divisions into tribes and demes, and task-specific work teams using such procedures as relevant expertise aggregation [Ober 2013].

Such processes of knowledge aggregation proved to be highly effective in enabling a group of otherwise disparate and heterogenous individuals, when acting as (or in the context of) an institution, to get the 'right' answer to a given question, in three ways: firstly, by providing incentives for knowledgeable individuals to pool their knowledge for the benefit of the group (these incentives did not have to be financial, but could be in the form of social capital (reputation) in an economy of esteem [Pettit and Brennan 2004]); secondly, by ensuring that the cost of communication was sufficiently low to overcome the transaction cost incurred by getting information from "where it was" to "where it needed to be"; and thirdly, epistemic sorting processes sifted not only false information from the true but also sifted information useful in a given context from the irrelevant.

2.1.2 Knowledge Alignment (KAlig). A distinctive feature of classical Athens was the number of public buildings and public events dedicated to the practice of government; for example public monuments and architecture created focal points, presence and visibility, combined with communal rituals (parades, religious festivals, sporting events) and civic buildings (e.g. gymnasia) fostered the growth of politically-relevant common knowledge, which in turn enabled the non-hierarchical implementation of a plan conceived by knowledge aggregation.

Having reached the 'right' decision by aggregating knowledge, processes of knowledge alignment ensured that this same group of individuals with a common interest in coordinating their actions 
could do so effectively. Athens/Athenians then achieved high levels of coordination between individuals and institutions by intermixing four epistemic mechanisms for accurate collective coordination. These are first choice, where one agent acts and the others follow in an alignment cascade; informed leader, where one agent deemed 'more informed' is designated the leader and the others follow his/her direction; rule-following, where each agent believes there is a rule, expects others' actions to conform to that rule, and so follows the rule itself; and commitment-following, where credible pre-commitments from each are required to ensure that all will act in unison (e.g turning up for a battle with a weapon and armour is a credible pre-commitment to join in the coming fight as opposed to turning up empty-handed).

2.1.3 Knowledge Codification (KCod). In classical Athens, the dynamic forms of knowledge that were employed in the processes of aggregation and alignment were captured, through the act of codification, in a text - a written law (nomos) or decree (psēphisma). In many cases that text was inscribed on a marble stele - literally "written in stone" - and publicly displayed.

Thus, having achieved a successful coordination by aligning common knowledge, the outcomes of both collective decision-making and collective coordination yield even greater benefit through processes of knowledge codification. This effectively creates an institutional 'memory' which can inform future behaviour of institutional members. In Athens, dynamic forms of knowledge used in the aggregation and alignment processes were codified in written laws or decrees. However, the Athenians managed to ensure that such codified knowledge was sufficiently stable to allow for confident planning, political engagement and civic education, but also sufficiently fluid to avoid ossification and allow introspective improvement by amendment through learning and innovation.

\subsection{KM Design Principles for Socio-Technical Systems}

Fast-forwarding from classical Athens to the digital transformation we see that innovations such as 'smart devices', IoT and analytics are bringing automation to the creation of data, information and knowledge and its representations in digital and algorithmic form. These can be perceived as, in effect, a resource commons, itself requiring self-governance and knowledge management. To leverage this duality of knowledge, as both an object and an instrument in its own self-management and self-modification requires some strategic mechanisms and related design principles.

In general, a collective action problem in a self-governing socio-technical system can be considered to be composed of three 'phases' (cf. Figure 1): collective decision-making where the members (of the collective) agree on a course of action supposed to solve the problem; collective coordination where the members perform individual acts intended to satisfy or contribute to satisfying this course of action; and collective memory where the members use the outcomes of their deliberation and action to update the selection and coordination processes themselves, and reference them in the resolution of subsequent collective action problems. Note the cyclic nature of the inter-dependence (dual nature of knowledge as resource and instrument) means that mechanisms for inspection, introspection and intervention are also part of knowledge codification.

Our proposal is that good self-governance depends on the extent to which the executives of governing procedures respect the rights and interests of all the stakeholders. This in turn depends on how useful knowledge is organised, with a particular emphasis on knowledge management processes for aggregation, alignment and codification, and how its duality is recognised.

Thus, a set of design principles for knowledge management processes applied to socio-technical systems, supporting the strategic mechanisms discussed, is presented in Table 2 [Pitt et al. 2017].

Principle 1 implies that knowledge management itself can be seen as a knowledge commons [Hess and Ostrom 2007], for which the characterisation of institutions for knowledge commons in computational form [Macbeth and Pitt 2015] is relevant. This characterisation identifies both 
Table 2. Design Principles for Knowledge Management Processes

1 Clear boundaries (as per Ostrom) between who is a citizen/participant in knowledge aggregation and alignment processes, and who is not.

2 Processes generally aim to lower transaction costs, and increase aggregate value of social cooperation.

3 Agreement on certain matters on which decisions must be made as being of common interest (e.g. security, welfare of all within widest boundary).

4 Clear line between common interest questions and factional or partial goods questions. Appropriate procedural rules for decision-making in each domain.

5 Common knowledge by citizens of substantive rules and of procedural rules for making new rules and revising existing rules.

6 Epistemic diversity among citizens, along with distributed social knowledge of locus of expertise and reliability of experts.

7 Procedural rules ensure that valuable, diverse inputs are recognised as such and taken up as appropriate. Filtering process for assessing what (and whose) information input is (and is not) relevant to each specific sort of question.

8 Processes serve as focal points/bright lines for collective action by citizens against violations and specify a hierarchy of possible punishments depending on seriousness of violation.

participants and their roles, i.e. associated powers with provisioning, appropriating, aggregating and modifying knowledge. In Principle 2 (as per Ostrom's observations about the relationship between monitoring rules and the behaviour they are intended to regulate), knowledge aggregation is enhanced if the barriers to participation are lowered by the cost of social interaction being factored into expected behaviour. Similarly, participation in the process itself creates externalities, often in the form of social capital in an economy of esteem [Brennan and Pettit 2004].

Principle 3 is concerned with ensuring that values, which might be implicit in the rules of governance, are assigned 'first class status' in knowledge management. Then the aim of Principle 4 is to avoid conflicting issues of constitutional significance (like citizenship, which requires procedural rules which imposes slow or careful deliberation) with quotidian concerns (such as resource allocation, which requires fast and efficient procedures for 'real-time' decision-making).

Principle 5 is the knowledge management's corollary to Ostrom's principle of self-determination (those affected by a set of rules participate on their selection, modification and application) [Ostrom 1990]. This can only meaningfully happen if the knowledge of collective choice is commonly shared (i.e. that those who should know about rules, do know about them). This is the same as with any other right: citizens' rights effectively exist only if the citizens know how and can afford to exercise those rights.

The considerations of Principles 6 and 7 on epistemic diversity, reputable sources of knowledge and expertise are intended to resist the manipulation, distortion and falsification of information, and minimise the effects of confirmation bias and the subsequent polarisation of opinion.

Finally, in Principle 8, as corollaries to Ostrom's principles of monitoring and graduated sanctions, tampering with the knowledge commons and knowledge management processes should be considered as sanctionable offences. 
Table 3. Perspective's roles in the resource allocation context

\begin{tabular}{ll} 
Perspective & Responsibility \\
\hline Operational & $\begin{array}{l}\text { Resource availability (generation, need, common-pool), re- } \\
\text { source transactions (demand, provision, appropriation) } \\
\text { Institution membership, role assignment, allocation policies, } \\
\text { rule enforcement }\end{array}$ \\
Knowledge Management & $\begin{array}{l}\text { Critical evaluation of Operational and Governance pro- } \\
\text { cesses, system reformation mechanisms from membership }\end{array}$
\end{tabular}

\subsection{KM Applied to Resource Allocation}

Self-organising a set of mutually-agreed rules (e.g. creation, selection, modification and application of the rules by the system participants) with respect to an implicit set of shared values has been an effective blueprint for successful (self-)governance, from the management of common-pool resources [Ostrom 1990], through deliberative assemblies [Robert and Robert 2011], and on to the 'democratic' nation state [Ober 2008]. Ostrom's design principles, and corresponding institutional analysis of knowledge commons [Hess and Ostrom 2007], have both been formally analysed computationally in [Pitt et al. 2012] and [Macbeth and Pitt 2015] respectively.

We therefore seek to extend these computational platforms to operationalise the Athenian system of knowledge management as a complementary perspective over an Ostrom-esque institutional governance. In fact, we are proposing a three-perspective architecture for self-governing sociotechnical systems: a lower 'operational' perspective, based on unbundled 'smart' devices and sensors (e.g. SmartMeters); a middle 'governance' perspective supporting self-organisation and collective action; and an upper 'knowledge management' perspective, implementing mechanisms for knowledge aggregation (e.g. social networking with incentives for sharing, low costs for communicating, participating, etc.), knowledge alignment (coordination mechanisms such as first mover-, leader- or rule-following, and credible pre-commitments), and knowledge codification (law as code [Lessig 2009]).

In this work, we consider the role of each perspective in a problem of common-pool resource allocation. As mentioned already, this is a collective action problem that requires effective knowledge management processes to be addressed satisfactorily.

To describe such a scenario, we consider an open system where a group of agents has access to a common resource that is both produced and consumed by them. There is also a common pool to which all agents have access, being able to deposit or withdraw resources from. By having this common pool as a public interface between agents, they are expected to participate on a collaborative process by defining personal actions on how individual resources and demands should be presented to the public and decide collective actions for managing the common pool to the group's benefit.

In this context, each perspective (operational, governance and knowledge management) can be distinguished as we identify their responsibilities when dealing with the collective action problem. A summary of each perspective's role is presented in Table 3.

From the operational perspective, brute facts regarding resource availability (amount of resources produced, the need for resources, resources available in the common pool) and resource transactions (an account of resources provided and appropriated by agents) are observed. Also, basic communication between agents (requests for resources and communication of allocation) is considered. 
However, the operational space is potentially exposed to intentional exploitation as agents might deliberately choose to misrepresent their requests for resources, making demands for more resources than they actually need, provide fewer resources than they have generated or appropriate resources that have not been allocated. Therefore, a governance approach is justified.

From the governance perspective, the resource allocation process is institutionalised, as agents receive specific roles (e.g. head, ruler, police) and membership is considered. Thus, institutions define rules for resource access (allocation policies) and sanctioning and regulatory policies are established.

Yet, even the presence of an institution does not necessarily guarantee that the system will be governed in a fair, non-tyrannical and sustainable way, as rulers are prone to corruption and regimes can be authoritarian.

Therefore, the knowledge management perspective is necessary to verify that values are being respected by the governance and operational perspectives. A knowledge management perspective for the resource allocation context should critically evaluate the ongoing processes, verifying which are the values being enforced and ultimately if fairness is achieved through the current resource allocation processes.

An effective knowledge management process would guarantee mechanisms through which all agents' opinions can be expressed and heard, and that rules are defined in a participatory manner. Also, eventual discrepancies between individual and general satisfaction should be examined to avoid privileges of a class in the resource distribution. Finally, in cases of bad, inefficient or unfair governance, there should be mechanisms that allow the reformation of both rules and rulers, enabling the emergence of adaptive self-organised systems.

In the following sections, we will consider experimental scenarios in order to investigate what effective knowledge management can be seen in practice, as we operationalise different KM design principles. Table 4 summarises the experiments presented, defining the information being considered as input, the outcome socially productive purpose, and describing the KM perspective role in the problem.

\section{FORMAL MODEL}

As a way to see some of the KM Design Principles presented in Section 2.2 operationalised, we propose a formal model able to abstract a general resource allocation problem and investigate and simulate different approaches to solutions.

A suitable scenario to explore concepts of justice and fairness in multi-agent resource allocation is the Linear Public Goods (LPG) game [Gächter 2006]. The game consists of a scenario where agents receive resources independently and can decide either to cooperate or not. This game is frequently used to examine the effects of free-riding [Hardin 2013] in provision and appropriation

We will use a variation of the original game, the LPG' game [Pitt and Schaumeier 2012]. This involves extended rules and features more diverse behaviour, especially non-compliant actions. The overall objective is to a group of $n$ agents to share an environment with scarce and depletable resources, ensuring that both they and the resources are sustained (ideally indefinetely).

The $\mathrm{LPG}^{\prime}$ game is played in a sequence of rounds, each of which is a step in time. At each timestep $t$, each agent $i$ is independently presented with an amount of resources available $\left(g_{i} \in[0,1]\right)$ and an individual need for resources $\left(q_{i} \in[0,1]\right)$. We fix the condition that $g_{i}<q_{i}$, which creates an economy of scarcity, which in turn forces agents to share their resources in order to satisfy their needs.

Each agent then declares their demand for resources $\left(d_{i} \in[0,1]\right)$ and also makes their provision of resources $\left(p_{i} \in[0,1]\right.$ and $\left.p_{i} \leq g_{i}\right)$. The sum of all agents' provisions form a Common Pool $(P)$ that can be distributed among all the agents. 
Table 4. Summary of experiments presented (expansion of Table 1), with emphasis to the available information considered, the socially productive purpose achieved and a brief description of how the KM perspective can be used in practice.

\begin{tabular}{|c|c|c|c|c|}
\hline $\begin{array}{l}\text { KM } \\
\text { Design } \\
\text { Princi- } \\
\text { ple }\end{array}$ & Algorithm & $\begin{array}{l}\text { Information } \\
\text { made available }\end{array}$ & $\begin{array}{l}\text { Socially } \\
\text { productive } \\
\text { purpose }\end{array}$ & Description \\
\hline 6 & $\begin{array}{c}\text { Voices of } \\
\text { Justice (VoJ) }\end{array}$ & $\begin{array}{l}\text { Individual } \\
\text { perception of } \\
\text { fairness }\end{array}$ & $\begin{array}{c}\text { Feedback of } \\
\text { system's } \\
\text { operation }\end{array}$ & $\begin{array}{l}\text { Diverse/divergent perceptions of } \\
\text { fairness are considered (KAgg) and } \\
\text { combined (KAlig), so a single global } \\
\text { metric of the quality of the resource } \\
\text { allocation can be defined. }\end{array}$ \\
\hline 7 & $\begin{array}{c}\text { Distributed } \\
\text { Distributive } \\
\text { Justice (DDJ) }\end{array}$ & $\begin{array}{l}\text { Legitimate } \\
\text { claims of } \\
\text { justice }\end{array}$ & $\begin{array}{l}\text { Decision of } \\
\text { resource } \\
\text { allocation } \\
\text { policies }\end{array}$ & $\begin{array}{l}\text { Beyond aggregating (KAgg) and } \\
\text { converging (KAlig) personal opinions, } \\
\text { codify (KCod) the knowledge in } \\
\text { concrete allocation rules that govern } \\
\text { how resources are distributed. }\end{array}$ \\
\hline 8 & $\begin{array}{l}\text { Flexible } \\
\text { Monitoring } \\
\text { and } \\
\text { Sanctioning } \\
\text { (FMS) }\end{array}$ & $\begin{array}{l}\text { Behaviour } \\
\text { towards norms } \\
\text { (compliance } \\
\text { and non- } \\
\text { compliance) }\end{array}$ & $\begin{array}{l}\text { Definition of } \\
\text { criteria for } \\
\text { rule } \\
\text { enforcement }\end{array}$ & $\begin{array}{l}\text { Aggregate knowledge (KAgg) of } \\
\text { compliance to rules (through } \\
\text { monitoring) and take informed } \\
\text { decisions on best rule enforcement } \\
\text { policy (KAlig, KCod) as response. }\end{array}$ \\
\hline
\end{tabular}

The Common Pool is then distributed (following certain criteria) by an agent designated as the group's head and each agent receives a nominal allocation of resources $\left(r_{i} \in[0,1]\right)$. Although the allocation is defined by the head, each agent decides the amount of resources that it will attempt to appropriate from the pool $\left(r_{i}^{\prime} \in[0,1]\right)$. Algorithm 1 outlines the described steps of the game.

When provision $\left(p_{i}\right)$, demand $\left(d_{i}\right)$ and appropriation $\left(r_{i}^{\prime}\right)$ are defined, it is possible to observe whether an agent is complying with the game or not. Compliant agents should provide what they have available $\left(p_{i}=g_{i}\right)$, demand just what they need $\left(d_{i}=q_{i}\right)$ and appropriate exactly what was allocated $\left(r_{i}^{\prime}=r_{i}\right)$.

This game formulation imposes a series of constraints to the players, that will be explored in the results sections. First, there is a fixed constraint that is the scarcity assumption, defining that agents produce less resources than they need $\left(g_{i}<q_{i}\right)$. Second, a physical (or brute) constraint guarantee that agents can not provide more resources than they have $\left(p_{i} \leq g_{i}\right)$, nor appropriate more resources than what is available $\left(\sum_{i} r_{i}^{\prime}<P\right)$. Finally, a conventional constraint defines that agents should provide all they have available $\left(p_{i}=g_{i}\right)$, demand just what they need $\left(d_{i}=q_{i}\right)$ and appropriate exactly what was allocated $\left(r_{i}^{\prime}=r_{i}\right)$; failure to comply with a conventional constraint defines an non-compliant behaviour and will be especially considered throughout the experiments.

The following sections will consider different scenarios, built on top of the LPG' game, where the design principles introduced in Section 2.2 can be used to guide solution mechanisms. In particular, we consider operationalisations of Principles 6, 7 and 8 (Sections 4, 5 and 6 respectively), which in turn will investigate:

- Voices of justice (VoJ) - Principle 6: Opinion formulation and diffusion;

- Distributive Justice (DDJ) - Principle 7: Allocation policies definition; 


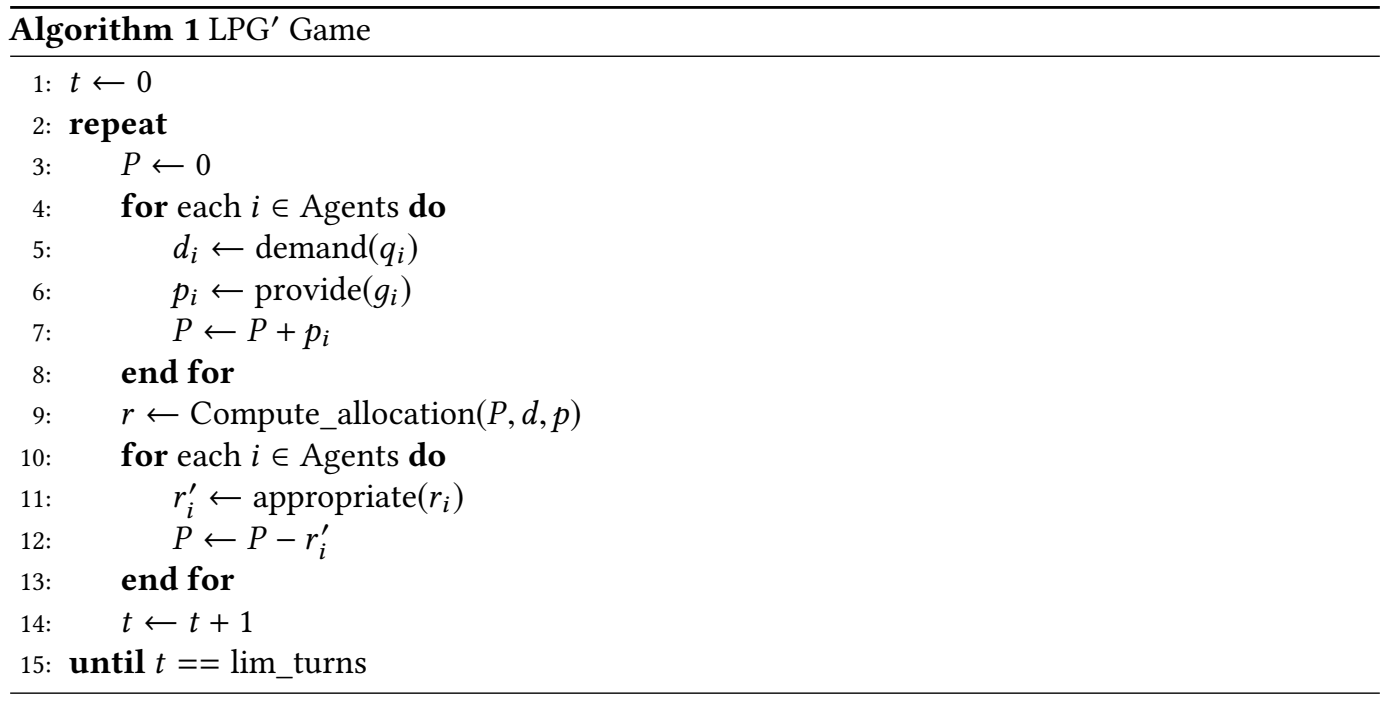

- Flexible Monitoring and Sanctioning (FMS) - Principle 8: Appropriate response for noncompliance;

Each section follows a common structure: (1) Motivation - a particular motivating scenario is presented, highlighting a specific collective action challenge that could justify the application of a corresponding KM principle; (2) Knowledge Management Principle - the KM principle terms are translated to the resource allocation context and goals are explicitized; (3) Experimental Setup although all three sections are resource allocation problems based on the $\mathrm{LPG}^{\prime}$ game, each situation brings particular aspects of collective decision-making, collective coordination and collective memory to be explored; (4) Operationalisation - a concrete algorithm formalises a strategy solution, operationalising the principle and exploring different KM strategies; (5) Results - from the formal model, simulations are performed and experimental results analysed for a variety of scenarios and research questions; (6) Reflections and Partial Conclusions - a discussion of the conclusions and reflections that can be drawn from the operationalisation of each principle.

The simulations performed on a bespoke multi-agent system simulator written in Erlang [Armstrong et al. 1993]. Although a single computer was used to run the simulations, the actor oriented paradigm of Erlang enables the execution of independent processes, so that the agent execution the execution model and the distributed algorithms would run in the same way in a networked system. Specific experimental parameters can be found in each section's results description. The code for all simulations is publicly available at https://gitlab.com/kurka/km-lpg.

\section{PRINCIPLE 6: VOICES OF JUSTICE}

Principle 6: Epistemic diversity among citizens, along with distributed social knowledge of locus of expertise and reliability of experts.

\subsection{Motivation}

A well-known aphorism in English law can be summarised as: "not only must justice be done; it must also be seen to be done". In part, the principle underpinning the aphorism can be traced back to one of the Roman ideals of natural justice, nemo judex in causa sua, literally that no-one should be a judge in their own cause. In other words, judges and judgements should be entirely neutral and objective, and not influenced by a personal stake or interest in a case or its outcomes. Moreover, 
not only should this principle be applied, but it should be verifiable and unquestionable that it has been applied.

We consider the implications of this statement for a policy of distributed distributive justice intended to deliver 'fair' resource allocation in open multi-agent systems. Although this policy aims to decentralise the process of administering distributive justice, in the absence of a non-repudiable distributed database such as that used in shared ledger technologies, it instead still relies on a designated agent occupying the role of a 'head', who is responsible for applying the algorithm that computes the resource allocation. The problem is that the agent, even if elected, may still be a recipient of resources itself; and even if that is not the case, it may still be influenced by, and hence favourable too, a clique or cartel of agents. Distributive justice may be done; but it is not being seen to be done.

The solution proposed here is to apply knowledge aggregation principles, through the use an information dissemination framework that is based on trust, social influence and opinion formation. Then, a process of knowledge alignment is executed, which allows multiple local processes to aggregate and converge on an overall feedback measure for the effectiveness of the system in delivering fair outcomes.

To form a global assessment of the system fairness based on individual accounts of the system's fairness, one has to consider that each agent might just have incomplete or inconsistent information. We therefore explore strategies to obtain reliable feedback in distributed systems, based on independent accounts of multiple agents. We consider a scenario where agents in a network, sharing a common-pool of resources, are subject to a pre-defined allocation policy (that can serve their demands or not). Then, from each agent's individual perspective, different (and possibly dissonant) claims are communicated and aggregated, resulting in an objective assessment of the general fairness of the allocations that have been made in the network. These perceptions can serve as a first indication of malfunctioning (or malfeasance) in the network, and produce a general mobilisation for change in the resource allocation protocol.

\subsection{Knowledge Management Principle}

We approach the problem of fairness perception utilising KM design principle 6, which is concerned with the preservation and appreciation of citizens' epistemic diversity. Diversity is a key element in multi-agent systems [Lewis et al. 2013], as it guarantees the consideration of multiple solution strategies, ensuring the exploration of an enhanced solution space for problems through distributed social knowledge.

It should be noted, however, that the system should also be capable of converging on a consensual solution by relying on an emergent locus of expertise, which arises from the information exchange and the recognition of experts' opinions.

For this specific resource allocation problem, we can directly translate some concepts from the original principle formulation. In our scenario, diversity of opinions can be found in the variety of agents' expression regarding the amount of resources they have or have not received.

Through information diffusion and convergence processes we can achieve distributed social knowledge and the clustering of opinions generates locus of expertise. Here, the exploration of different network topologies can also be considered as a factor to enhance or reduce locus of expertise.

Finally, in order to weight opinions and recognise expertise in debatable affairs, the formulation of trust and affinity metrics can be used, guiding the influence and information diffusion processes. 


\subsection{Experimental Setup}

Consider a group of $n$ agents, with connections defined by a topological graph $G$. Each agent performs independent activities over time and requires resources to fulfil its tasks.

At a specific time (turn) $t$, an amount of resources $P(t)$ is made available to the group and should be shared among its members. Each agent's demand is calculated independently and is denoted $d_{i}(t)(i \in 1, \ldots, n)$. Suppose the amount of resources per turn is less than the total demand $\left(P(t)<\sum_{i} d_{i}(t)\right)$, creating a scenario of scarcity where not all agents are able to receive enough resources every turn.

Given the resources available and the agents' demands, an allocation policy is defined and each agent receives an individual attribution $\left(0 \leq r_{i}(t) \leq d_{i}(t)\right)$, until the whole common-pool of resources is distributed $\left(\sum_{i} r_{i}(t)=P(t)\right)$. The allocation policy can be considered fair or not, depending on the amount of resources given to the agents over time.

Each agent can maintain a measurement of perceived fairness over time $\left(\phi_{i}(t)\right)$, which is influenced both by its individual and local perceptions of how resources are being allocated. An agent can also observe its neighbours' perceived fairness. Individual perceptions of fairness can be grouped, resulting into an collective perceived fairness $\Phi(t)$, that can be used as a metric for general satisfaction

Given a group engaged in repeated rounds of resource distribution and agents' personal opinions and interactions, how can it be ensured (be sure) that an allocation is 'fair'?

\subsection{Operationalisation}

The framework proposed as a solution to this problem, consists in using individual subjective assessments of fairness, in order to achieve a collective objective sense of fairness.

This use of individual assessments, expressed by each agent to its neighbours, brings at least three benefits. First, it allows an inherent decentralisation of the feedback process, as subjective impressions are formed independently and autonomously. Second, as many witnesses corroborate independently to the acknowledgement of a fact, the reliability of the process is increased (related to the preponderance of evidence). Third, it gives voice to "regular" individuals that might be subject to an unfair treatment and possibly would not have their opinion considered in centralised evaluations.

Nevertheless, this approach does also presents some challenges. First, how should we deal with malicious agents that try to manipulate its individual opinions, in order to misguide the general opinion (and possibly benefit itself)? Second, how should opinions be weighted, in cases where there is discordance of opinions?

The solution proposed is structured as follows. At every turn, after the common-pool of resources is divided among agents in a group, four main steps take place in order to produce an output of general perceived fairness:

(1) Opinion formation - agent opinions are formulated, based on individual experience;

(2) Trust - agents observe their environment and, through comparison, update the trustworthiness of their neighbours;

(3) Influence - agents communicate and diffuse opinions through their social influence;

(4) General fairness assessment - the multiple opinions converge to a single assessment on the system's fairness.

The following sub-sections elaborate on each of these steps in turn.

4.4.1 Opinion Formation. Each individual, in light of the amount of resources it has received over time and the amount of resources it has demanded, can elaborate its own subjective opinion on the fairness of an allocation method. 
There are many ways of doing this measure. A simple measurement, for example, would be the percentage of turns on which an individual's demand is granted:

$$
\phi_{i}(t)=\frac{\sum_{s=1}^{t} \mathbb{1}\left\{r_{i}(s) \geq d_{i}(s)\right\}}{t}
$$

However, a metric that can take into consideration the temporal evolution of the distribution (and not just the average) can also reveal other interesting aspects of the (un)fairness of a policy. A useful metric that fits this purpose, proposed in [Pitt et al. 2014] is the satisfaction index given by:

$$
\phi_{i}(t)= \begin{cases}(1-\alpha) \cdot \phi_{i}(t-1)+\alpha & \text { if } r_{i}(t) \geq d_{i}(t) \\ (1-\beta) \cdot \phi_{i}(t-1) & \text { if } r_{i}(t)<d_{i}(t)\end{cases}
$$

where $\alpha$ and $\beta$ are respectively positive and negative reinforcement rates $(\alpha, \beta \in[0,1])$ and can be adjusted to represent the level of optimism/pessimism of individual agents with respect to the system conditions.

4.4.2 Trust. Having formulated their personal opinions, agents then start to exchange opinions with each other, in order to compare their assessments. This comparison is important as the knowledge of each individual is limited to its context and, by observing its neighbours, information of the environment of more distant areas of the network can be obtained and contribute to a more qualified impression of what is happening in the network.

Therefore, a mechanism for updating an individual opinion, given its contact to different opinions should be developed. However, before exploring the transmission of information, one important issue should be considered: how to accommodate and reconcile multiple independent accounts.

In scenarios where there is a discrepancy between neighbours' opinions, there should be ways of weighting the relevance of each received account. Considering that biased and non-compliant behaviour can be a feature of social interaction, each individual should also protect itself from false accounts and find impressions as close to the truth as possible.

Effectively, what is being done here is the epistemic sorting process investigated in [Ober 2008] and mentioned in Principle 6. In the Athenian case, this process was used so citizens could actually learn how and who to listen upon a process of collective decision-making.

What is crucial for this epistemical sorting process is the notion of trust. Castelfranchi and Falcone [Falcone and Castelfranchi 2001a,b] introduce this concept in multi-agent systems; in their formalisation, a framework is built where agents decide to rely or not on its peers by evaluating two main factors: (a) a subjective confidence, based on the analysis of past interactions (sociocognitive aspects) and (b) the direct benefits that a relationship of trust can bring (socio-economic aspects). Therefore, trust is a function of not only past experiences, but also the personal benefits in interacting with an agent, even if it is known that he/she is cheating.

Another concept, discussed in [D'Agostino and Sinigaglia 2010], is the use of scoring rules as a way of measuring agents' reputation and assuring that agents' claims are consistent to their actual experiences. A scoring rule is a function, $S\left(\phi_{i}(t), R_{i}, D_{i}\right)$, that takes into consideration an agent's claimed opinion $\left(\phi_{i}(t)\right)$ and the history of resources received $\left(R_{i}=\sum R_{i}(t)\right)$ and demanded $\left(D_{i}=\sum d_{i}(t)\right)$, returning an index. The function is defined in such a way that if the agent's opinion is in accordance with its perception of reality, them the index returned is high; if an opinion is not in accordance with its context, then a low index is returned.

Therefore, taking these two concepts into consideration (trust and scoring rules), we can define a metric that computes the trust $\left(T_{i, j}\right)$ of agent $i$ in $j$. 
Initially, a metric is defined to determine the divergence of opinions in a given time. The accordance index $\tau_{i j}(t)$ is a value in the range $[0,1]$, representing the level of "agreement" between two individuals ( $i$ and $j$ ) at timestamp $t$.

An initial simple definition of $\tau$ can be given solely by the difference of opinions of $i$ and $j$ :

$$
\tau_{i j}(t)=1-\left|\phi_{i}(t)-\phi_{j}(t)\right|
$$

Eq. 3 can be improved with more information, considering not only agent $i$ 's perception, but also its context given by its neighbours' opinions (the neighbourhood of $i$ denoted $N(i)$ ):

$$
\begin{aligned}
\tau_{i j}(t) & =1-\left|\bar{\phi}_{N_{i}}(t)-\phi_{j}(t)\right| \\
\text { where: } \quad \bar{\phi}_{N_{i}}(t) & =\frac{1}{|N(i)|+1} \sum_{n \in N(i)-\{j\}+\{i\}} \phi_{n}(t)
\end{aligned}
$$

Extending $\tau$, we can specify a function where small differences of opinions are overlooked and bigger differences are amplified. One example is the logistic function defined as:

$$
\tau_{i j}(t)=1-\frac{1}{1+e^{-k\left(\left|\bar{\phi}_{N_{i}}(t)-\phi_{j}(t)\right|-\epsilon_{0}\right)}}
$$

where $k$ is a coefficient defining the steepness of the transient in the logistic curve and $\epsilon_{0}$ is the midpoint of the curve, representing a threshold for the difference of opinions before the accordance goes rapidly to 0 . The shape of the logistic function means that minor differences only have a minimal impact on the affinity, while a major divergence has a larger impact on it.

Note that all definitions of $\tau$ extract elements of [Falcone and Castelfranchi 2001a,b], as individuals that agree with each other tend to trust in each other, turning trust into a function of reinforcement of personal opinions and self-benefit.

However, aspects concerning individuals' past experiences and agents' reputation should also be considered. In the light of scoring rules, we then formulate the definition of Trust $T_{i j}(t)$ as a function of previous trust assessments $\left(T_{i, j}(t-1)\right)$ and the current accordance $\left(\tau_{i j}(t)\right)$ :

$$
T_{i j}(t)=(1-\gamma) \cdot T_{i j}(t-1)+\gamma \cdot \tau_{i j}(t)
$$

where $\gamma \in[0,1]$ is a defined reinforcement rate and $T_{i j}(0)=1$, meaning that agents consider each other trustful a priori, before empirical evidence.

Note, however, that if agents $i$ and $j$ are not connected in the network, their accordance index $\tau_{i j}$ and trust $T_{i j}$ are equal to 0 , as no knowledge is shared.

4.4.3 Influence. Having the personal opinions $\phi$ and the trust assessments $T$ we can model the evolution of opinions under social influence. The idea is that, when an agent is in a context of divergence of opinions, it will update his/her own initial opinion, using his/her neighbours' opinions and reputations. In [DeGroot 1974], an interactive process is used to propagate opinions subject to trust.

Firstly, at each timestamp, a trust matrix $T$ is built, formed by values of $T_{i j}(t)$ for all combinations of individuals $i$ and $j$. Every row of $T$ is normalised by its sum, so the line $i$ in matrix $T$ has a probability distribution with sum 1, representing the trust distribution of agent $i$ in all group members.

Then, given a vector with all opinions, $\phi(t)=\left[\phi_{1}(t), \ldots, \phi_{n}(t)\right]$, by multiplying the matrix $T$ over vector $\phi$ multiple times, we obtain the updated opinion vector $\phi^{\prime}$ :

$$
\phi^{\prime}=T^{K} \phi
$$


where $K$ is a number of iterations, generally large enough $(\geq 100)$ to allow convergence.

4.4.4 General Fairness. Finally, in order to compute a general measure of the fairness, we compute the simple average of all individual opinions (after influence process):

$$
\Phi(t)=\frac{1}{n} \sum_{i} \phi_{i}^{\prime}(t)
$$

All the steps described in this section and the final equations used are summarised in Algorithm 2.

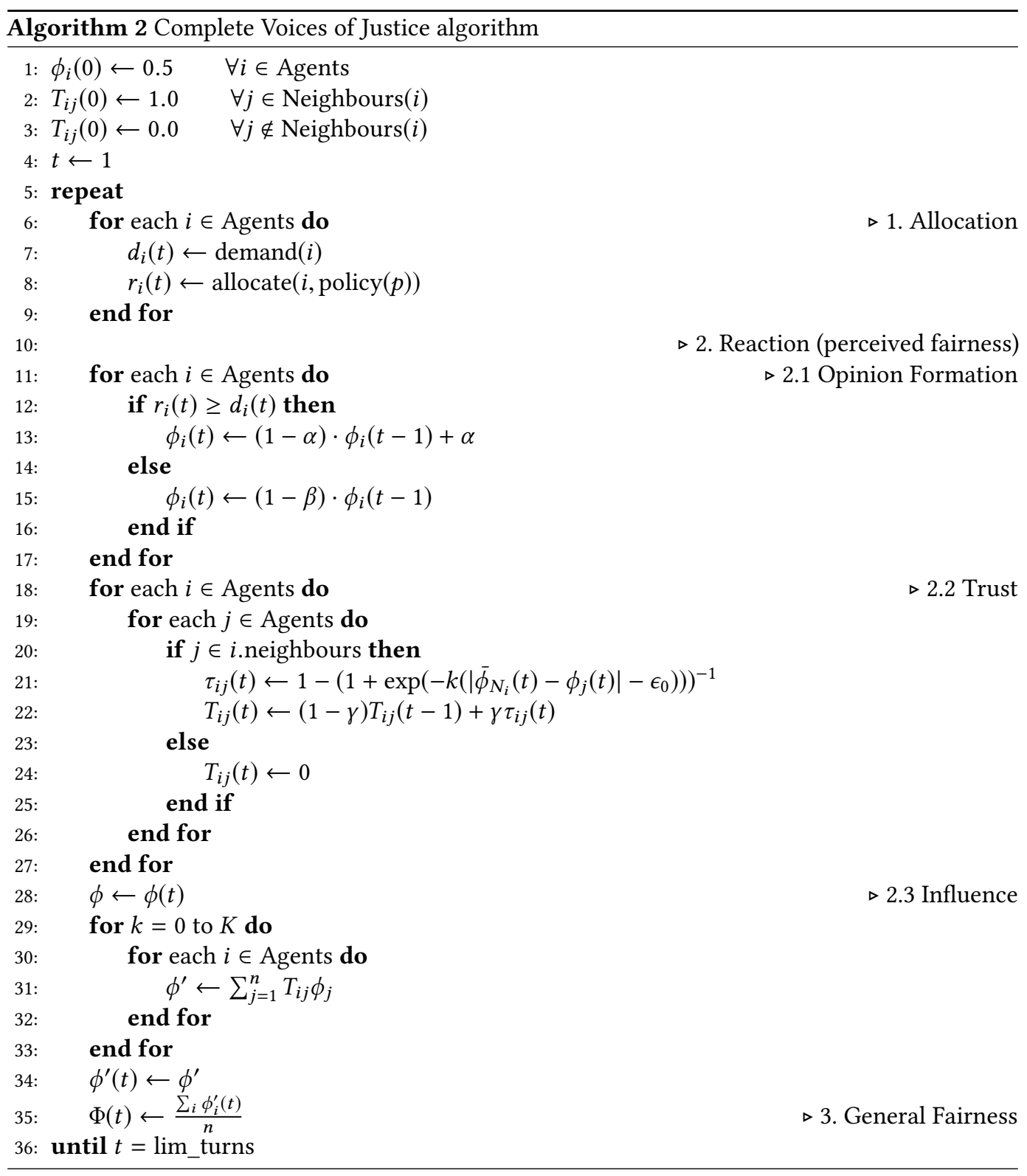




\subsection{Results}

We design a series of simulation experiments to evaluate the efficiency and effectiveness of Algorithm 2 (VoJ), where in particular we consider the following questions:

- Coherence - can VoJ identify and distinguish between fair and unfair allocation schemes?

- Robustness - is VoJ able to avoid the influence of malicious agents trying to propagate false information?

- Resilience - how does VoJ function under different topologies and topology changes?

The open parameters from the operationalisation section are defined as follows. We consider a population of $n=30$ agents. We consider that in every turn, agents have a fixed demand of $d_{i}(t)=1.0$ for resources, but only half of the total demand is made available per turn: $P(t)=\frac{n}{2}$. The satisfaction index parameters (Eq. 2) were defined as: $\alpha=\beta=0.1$. The accordance index (Eq. 5) used $k=20$ and $\epsilon_{0}=0.2$ and trust reinforcement rate (Eq. 6) as $\gamma=0.1$. In the influence phase, opinions are updated until convergence or at most $K=100$ iterations.

4.5.1 Coherence. The first experiment consisted of testing different allocation schemes and in which ways the network reacted to fair and unfair allocations. We tested four different allocation strategies, as described below:

- rotation: alternate users in order, serving the demand of a subset of agents each turn and ensuring that all agents are served in the same frequency over time;

- clique first: serve first the demand of a fixed 'elite' group (or 'cartel') and then (if there are any remaining resources) the others in random order;

- random order: draw an order of service every turn and fulfil demands in order, until there are resources left;

- ration: divide the turn's resources among all agents equally, giving the same share to all individuals (independently of their demands).

We can say that both "rotation" and "random order" are fair allocations, as on average every agent receives the same amount of resources over time. The "clique first" strategy is unfair, as it benefits a closed group, to the detriment of others. Although "ration" shares equally resources among all agents, as it does not allocate enough resources to satisfy agents' demands, individuals subject to this allocation scheme have low satisfaction, revealing that it is also an unfair scheme.

Figure 2 shows a regular ring lattice network, with neighbourhood equal to 4 , and the values of personal perceptions $(\phi)$ and collective perceived fairness $(\Phi)$ after 500 turns $(t=500)$ for the four allocation schemes. For each allocation case, the left-hand side presents values for $\phi$ before the propagation (influence) step and the right-hand side shows the propagated values $\left(\phi^{\prime}\right)$.

We see that in all four cases, the propagated opinions $\phi^{\prime}$ converge to approximately the same value, showing that there is agreement on the general fairness. Also, we see that the network can identify correctly the fair allocations, as seen in the values of $\Phi$ obtained in each scenario. As the total demand is the double of the available resources $P$, the baseline value for a fair $\Phi$ is equal to 0.5 .

It is particularly interesting to note the case of clique allocation (upper right) where, although there is a big range of opinions, $\phi^{\prime}$ converges to a low value (0.4), revealing the unfairness of the allocation. It is also worth noticing that, in such scenarios, even highly satisfied agents realise that their opinion is not appropriate and, by losing their trust in themselves, accept their unhappy neighbours opinions' as their own.

4.5.2 Robustness. In a second experiment, we investigated the effects of agents giving false feedback. We simulated a scenario where a fair allocation takes place (rotation), but regardless of the fact that every agent receives the same amount of resources over time, a group of misbehaving agents always give a negative feedback. 
Rotation

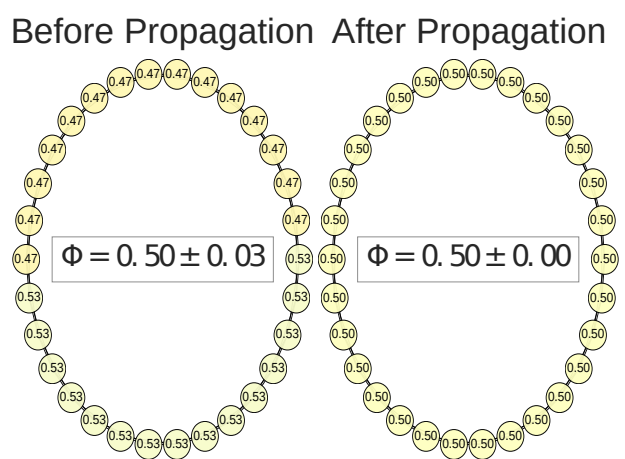

Random Order

Before Propagation After Propagation

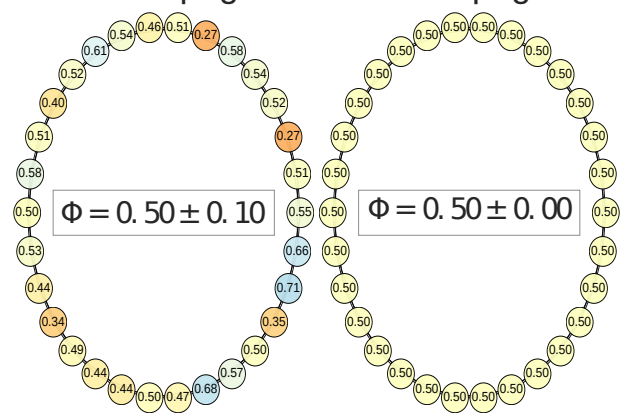

\section{Clique First}

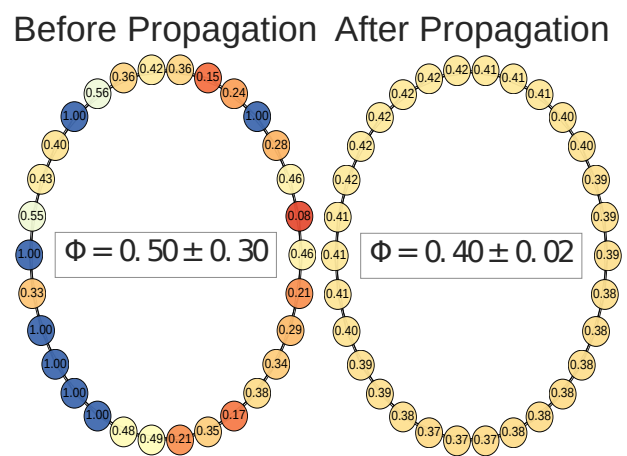

Ration

Before Propagation After Propagation

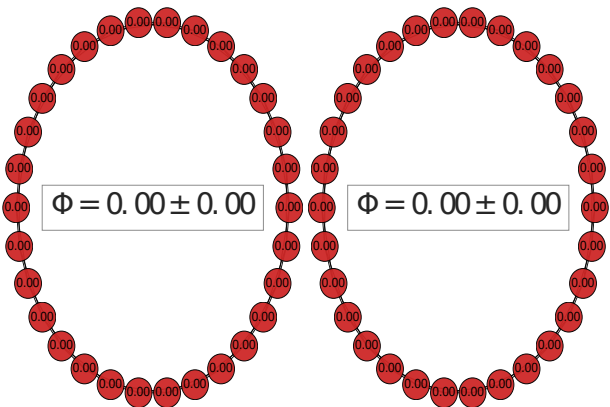

Fig. 2. Agent's opinions for different allocation policies, after 500 iterations, with and without trust propagation.

Figure 3 presents a simulation using the same topology as before with 5 non-compliant agents (out of 30).

Figure 3a shows the average opinion $(\Phi)$ of both the 'honest' and 'dishonest' agents before propagation, demonstrating the difference of perception of both groups. We then see in Figure $3 b$, shows that when trust and influence are applied the difference of opinion is suppressed and $\Phi$ converges to the same value expressed by the compliant group.

This shows that our trust framework is capable of discarding unreasonable opinions, with coherent ones prevailing.

4.5.3 Resilience. In a third experiment, we explored the impact of the network topology on the information diffusion and influence mechanisms, so we can evaluate the resilience of $\mathrm{VoJ}$ in different configurations. Thus, we examined the performance in two different network topologies: small world [Watts and Strogatz 1998] (Figure 4a) and random graph [Erdös and Rényi 1959] (Figure 4b). In each case, a fair (rotation) and an unfair (clique first) allocation scheme was tested and values of propagated opinions $\left(\phi^{\prime}\right)$ were reported.

We see that the algorithm achieves satisfactory results in different topologies. However, when the results are compared to the ones obtained in the ring network topology (previous experiments), we notice that the convergence of the small-world network is superior, while the random network presents more divergence of values. 


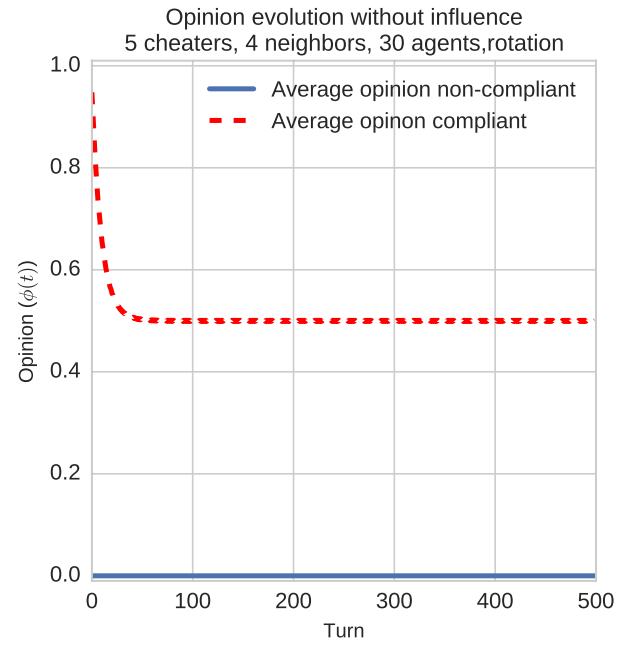

(a) Mean opinions, without influence.

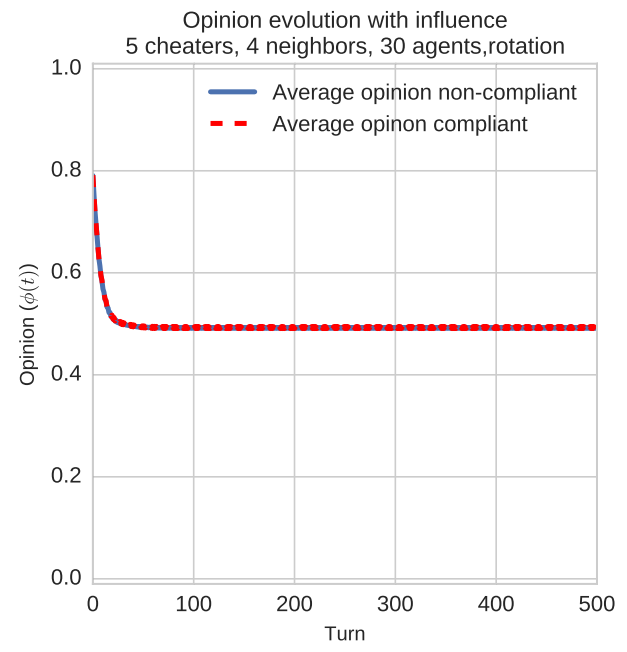

(b) Mean opinions, with influence.

Fig. 3. Simulations with the presence of a cheating agents that are always unsatisfied with the allocation, no matter how fair it is.

This can be explained by the fact that small-worlds can be seen as a derivation of ring lattices [Watts and Strogatz 1998], in such a way that it preserves the regularity of most neighbourhood connections, but also adds more distant connections that can help in the propagation of information during the influence phase. The random network, on the other hand, presents more irregularities in its connections, possibly hindering the communication and the achievement of consensus.

\subsection{Reflections and Partial Conclusions}

The formal framework presented in this section brings important insights for the achievement of distributed social knowledge in scenarios of epistemic diversity, as expressed by KM design principle 6.

In a scenario of multiple competing claims, such as the case of personal evaluation of fairness of a resource allocation system, we show that such processes of knowledge aggregation and alignment gives voice to multiple agents, but at the same time finds consensus from the appreciation of diverse opinions.

This encourages the design of socio-technical multi-agent systems in which participants feel heard and included, motivating their participation and integration in the system. Besides that, the distributed nature of the framework that enables decentralised and independent computation of an allocation fairness might increase the participant's confidence in the system, compared to centralised black-box strategies.

Our experimental results demonstrate that such a strategy is able to find coherent and reliable outcomes, even within initial divergence of opinions, correctly distinguishing between fair and unfair allocations. This reliability is a direct consequence of the knowledge management process proposed, through the trust and influence model formulated, that enables the emergence of a locus of expertise as trustworthy experts are endorsed.

The reliability of the outcomes is an important element when considering the possibility of malicious behaviour by propagation of false information. Our experiments show that the model is 

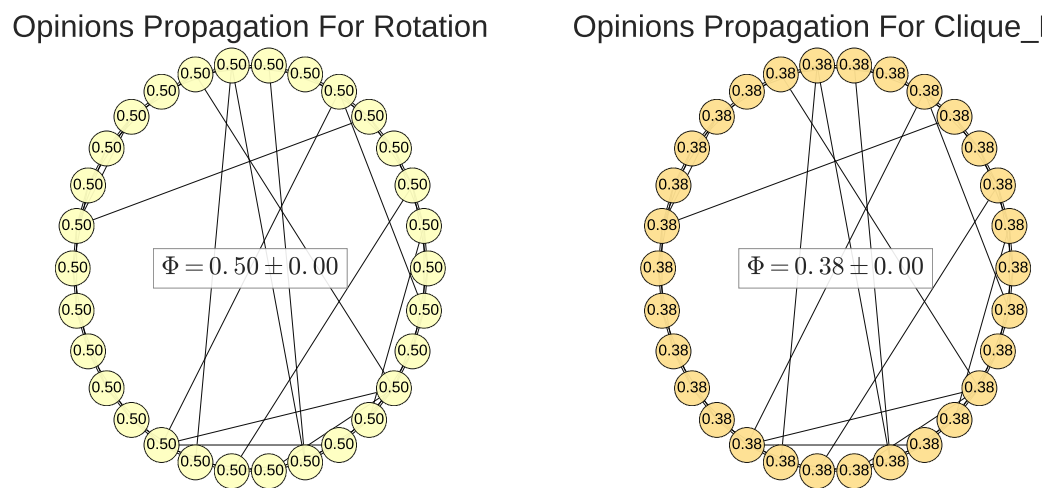

(a) Information diffusion in small-world network
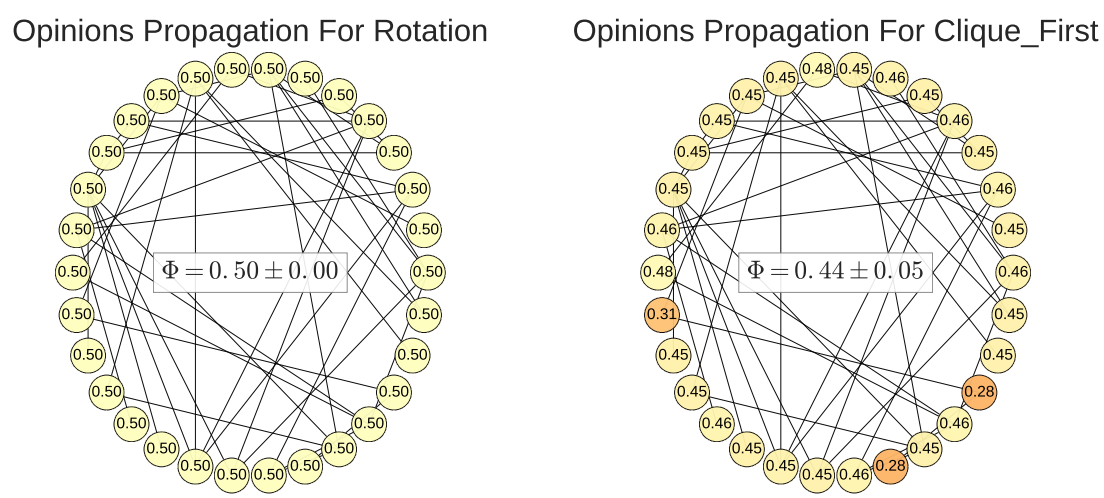

(b) Information diffusion in random network.

Fig. 4. Result of information diffusion in different topologies, for two allocation schemes

robust and resilient to different scenarios, being able to identify and even exclude (through lack of trust) dysfunctional behaviour.

\section{PRINCIPLE 7: DISTRIBUTED DISTRIBUTIVE JUSTICE}

Principle 7: Procedural rules ensure that valuable, diverse inputs are recognised as such and taken up as appropriate. Filtering process for assessing what (and whose) information input is (and is not) relevant to each specific sort of question.

\subsection{Motivation}

In scenarios of abundance of resources, the problem of resource allocation in networks can be solved simply by the creation of an efficient system of resource distribution, that contemplates every individual requesting assets. In this case, the optimal solution is found when costs of communication or transportation are minimised and the system can run with the lowest possible cost.

However, in situations where there are insufficient resources to satisfy the whole network demand (i.e. a context of scarcity), alternatives approaches to the problem are required which focus in other areas besides, for example, cost minimisation. 
It is in this context that the concept of computational justice [Pitt et al. 2015] was introduced, whose objective is to evaluate the 'correctness' of the outcome of algorithmic deliberation with respect to some qualitative criteria (e.g. fairness).

Previous work [Pitt et al. 2014, 2015] shows that, by pursuing computational justice, systems are able to self-organise sustainable strategies for resolving a resource allocation problem with regard to fairness. In particular, the strategies do not seek only to maximise individual resources, but also aim at other qualities, such as "fairness", "appropriateness" and "acceptability".

Now, we extend this previous work, by applying it in support of the operationalisation of KM principle 7 so we can continue to find fair solutions for the resource allocation problem, but in a decentralised and distributed way, through collective decision-making, coordination and codification. We extend knowledge management processes to include executive action, creating a communication structure that enable agents to take informed and conscious decisions.

When measuring the fairness of a distribution of resources (either in real or artificial contexts), there is a tendency to prioritise the utilitarian aspect of the distribution, i.e. the quantity of individuals receiving resources and the amount allocated, as it is the most immediately quantifiable and measurable. Nicholas Rescher, however, when exploring the issue of distributive justice [Rescher 1966], realised that just the utilitarian metric alone is not enough to evaluate all the different aspects of the fairness in a system. Instead, he proposes seven different canons of distributive justice, each of which capture a different criterion for distributing rewards and punishments.

Each canon relates to a different principle of fairness, including equality, but also adding aspects of needs, productive contribution, efforts and sacrifices, socially useful services, supply and demand, and merit or achievements. Rescher argues, then, that these different aspects of justice should be considered in conjunction because each one in isolation was inadequate as the soul arbiter of distributive justice.

Therefore, the theory presented by Rescher provides the basis for investigating fairness in environments of resource distribution and collective action. The method is to first identify which of the canons are operational in the scenario, and express them by one or more of (what Rescher called) legitimate claims. Then, for each legitimate claim a formal metric is defined for computing the allocation order of this claim. Finally, we specify a process for accommodating multiple legitimate claims and reconciling them if they are in conflict.

Our objective is, therefore, to apply this methodology to the LPG' game. Accordingly, we develop a distributed strategy for fair resource allocation, so that agents most in need have priority in the allocation and non-compliant agents are discouraged or lose priority.

\subsection{Knowledge Management Principle}

While KM design principle 6 was effectively concerned with knowledge aggregation, in how to consider diversity of positions and knowledge, principle 7 explores the knowledge alignment and knowledge codification aspects as it discusses how the diversity of knowledge can be filtered through procedural rules and knowledge can be selected and chosen to answer and decide specific questions. Although diversity of opinions is still a relevant factor, the processing, selection and convergence of information is necessary to enable course of action.

In our current scenario the course of action being considered is the actual allocation of resources. We require a strategy that is able to, in the midst of plurality of opinions and requests for distribution of resources, coordinate and codify allocation rules that should be used to serve agents. Thus, the procedural rules being applied are those defined by the resource allocation policy, which determines the priority order of allocation. 
Given the subjectivity of the task, we need to consider and recognise the value in different demands for resources and its appropriateness. For this, we use Rescher's legitimate claims, as a way to guarantee plurality and representaton of agents' expressions.

Finally, in order to take action and distinguish among different opinions in order to converge in a practical allocation policies, we sort and filter relevant information by using the concept of trust, reputation and affinity.

\subsection{Experimental Setup}

Consider a group of $n$ agents, with connections defined by a graph $G$. Over time agents produce, provide, need, demand resources and use a common pool $P$ for distributing resources, according to the $L P G^{\prime}$ game rules.

The goal of this experiment is the definition of an allocation policy for the distribution of the resources of the common pool. In practical terms, an allocation policy is a sequence of size $n$, defining the order in which agents will access the common pool and the amount of resources allocated per agent $\left(r_{i}\right)$.

The allocation policy is defined every round and is deliberated with the participation of all agents of the group, through information exchange, expression of opinions and voting mechanisms. An agent with the special role of 'head' is responsible for defining and disclosing a final decision at the end of the process.

As stated in the rules of the game, agents can choose to comply or not with the allocation policy in place and decide whether to cooperate with the common pool (by providing resources or demanding just its real need).

As a way of evaluating the quality of the resource allocation process of a group of agents, at every turn we compute three metrics that give different perspectives on the allocation results.

First, we consider agents' utility Ut, as function of the resources transacted:

$$
U t_{i}= \begin{cases}a\left(q_{i}\right)+b\left(R_{i}-q_{i}\right) & \text { if } R_{i} \geq q_{i} \\ a\left(R_{i}\right)-c\left(q_{i}-R_{i}\right) & \text { otherwise }\end{cases}
$$

$$
\text { with } R_{i}=r_{i}^{\prime}+\left(g_{i}-p_{i}\right)
$$

$R_{i}$ is the total resources accrued by an agent $i$ at the end of a round and $a=2, b=1$ and $c=3$, representing respectively the relative utilities of getting resources that are needed, getting resources that are not needed, and not getting resources that are needed.

Second, as the game progresses, we count the number of remaining compliant (r.C) and noncompliant agents $(r . N C)$, as an indication of the behaviours present in the game.

Finally, as a way of evaluating the fairness of the allocation scheme, we use the Gini index [Gini 1912] over the distribution of the ratio between received and demanded resources $\sum r_{i} / \sum d_{i}$. An index of 100 represents a completely unequal distribution, while 0 represents total equality.

\subsection{Operationalisation}

We aim to develop a strategy of fair resource allocation, so that agents in need have priority in the allocation and non-compliant agents should be discouraged and lose priority.

Every turn, as the common-pool of resources is created, an allocation order is computed as the outcome of the following steps:

(1) Opinion Formation - using a formalisation of Nicholas Rescher's theory of distributive justice, agents elaborate personal perceptions of the environment's fairness, using different metrics; 


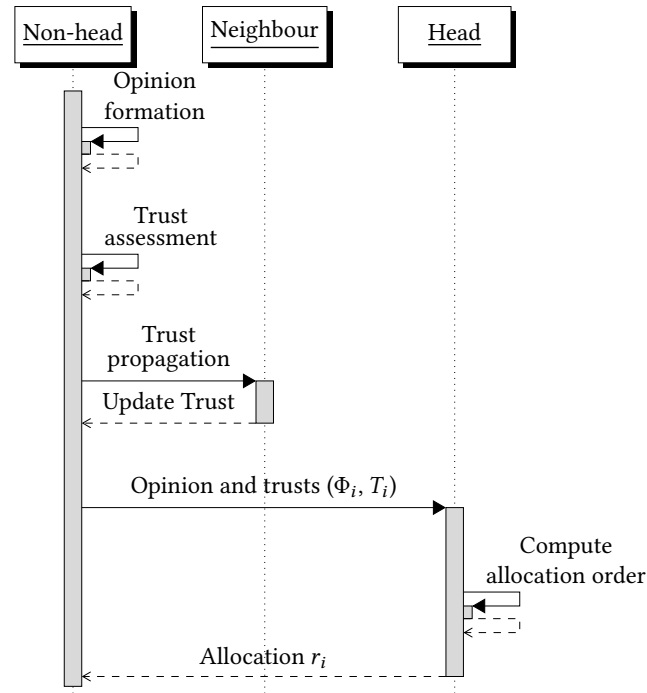

Fig. 5. Sequence diagram for distributed distributive justice allocation process framework, showing actions and actors involved in each step.

(2) Trust Assessment - individual opinions of fairness are compared to the opinions of other agents;

(3) Trust Propagation - agents' reputations are measured through a mechanism of trust and belief propagation;

(4) Reputation Based Allocation - by combining agents' claims and reputations an allocation order is computed, deciding how agents will be served.

A scheme of the steps of the strategy, with the parties responsible for each part can be seen in the sequence diagram of Figure 5.

5.4.1 Personal opinion formation - legitimate claims of justice. The first step of our algorithm is the formulation of personal opinions, by the agents, of the fairness of the processes taking place in the network.

This process builds on the formalisation of Rescher's legitimate claims specified in [Pitt et al. 2014]. However, there are at least four key differences from the previous work being introduced here: less waiting, no weighting, no voting and diminish reliance on the central role of head [Kurka and Pitt 2016a].

Given a turn $S$ of the LPG' game where all six variables availability $(g)$, need $(q)$, provision $(p)$, demand $(d)$, allocation $(r)$, appropriation $\left(r^{\prime}\right)$ were instantiated, we can measure merit according to the metrics presented in Table 5.

Thus, an individual's opinion vector $(\Phi)$ is defined by a vector containing the set of claims computed every turn, using the equations from Table 5.

$$
\Phi_{i}(t)=\left\{\phi_{i}^{1}, \phi_{i}^{2}, \phi_{i}^{3}, \ldots, \phi_{i}^{8}\right\}=\left\{\phi_{i}^{c}: c \in|C|\right\}
$$

It is also possible to define an aggregated opinion index $(\phi)$, as the sum of every claim value. Although all claims are in the range $[0,1]$, some of them represent maximum fairness when its value is equal to 1 (canons of equality and needs) and others when its value is equal to 0 (canons 
Table 5. Legitimate claims of justice. Extracted from [Pitt et al. 2014]

\begin{tabular}{|l|l|}
\hline \multirow{3}{*}{ Canons of equality } & $\phi_{i}^{1}(S)=\frac{\sum_{t=0}^{S} r_{i}(t)}{S}$ \\
\cline { 2 - 3 } & $\phi_{i}^{2}(S)=\frac{\sum_{t=0}^{S}\left(r_{i}(t)>0\right)}{S}$ \\
\cline { 2 - 3 } & $\phi_{i}^{3}(S)= \begin{cases}(1-\alpha) \cdot \phi_{i}^{3}(S-1)+\alpha \quad \text { if } r_{i}(S) \geq d_{i}(S) \\
(1-\beta) \cdot \phi_{i}^{3}(S-1) & \text { if } r_{i}(S)<d_{i}(S)\end{cases}$ \\
\hline Canon of needs & $\phi_{i}^{4}(S)=\frac{\sum_{t=0}^{S} d_{i}(t)}{S}$ \\
\hline Canon of productivity & $\phi_{i}^{5}(S)=\frac{\sum_{t=0}^{S} p_{i}(t)}{S}$ \\
\hline Canon of effort & $\phi_{i}^{6}(S)=S$ \\
\hline Canon of social utility & $\phi_{i}^{7}(S)=\frac{\sum_{t=0}^{S} I(h e a d(t)=i)}{S}$ \\
\hline Canon of supply and demand & $\phi_{i}^{8}(S)=\frac{\sum_{t=0}^{S} I\left(p_{i}(t)=g_{i}(t) \wedge d_{i}(t)=q_{i}(t) \wedge r_{i}^{\prime}(t)=r_{i}(t)\right.}{S}$ \\
\hline
\end{tabular}

${ }^{1}$ Eq. 3 is also called satisfaction index and $\alpha$ and $\beta$ are respectively positive and negative reinforcement rates that can be adjusted to represent the level of optimism/pessimism of individual agents as the system changes.

${ }^{2} \mathcal{I}$ is the indicator function, being equal to 1 if its argument is True, equal to 0 otherwise

of productivity, effort, social utility and supply and demand). Therefore, in order to allow the aggregation of all claims, some of them are negated as in Equation 11.

$$
\begin{gathered}
\phi_{i}(t)=\sum_{c} \frac{1}{|C|} \cdot \phi_{i}^{c^{\prime}}(t) \\
\phi_{i}^{c^{\prime}}(t)= \begin{cases}\phi_{i}^{c}(t) & \text { if } c \in\{1,2,3,4\} \\
1-\phi_{i}^{c}(t) & \text { if } c \in\{5,6,7,8\}\end{cases}
\end{gathered}
$$

Note that Equation 11 is an extension of the previously defined 2. The first definition just considered the canon of equality on its formulation, so we expect that the current version could have more explanatory power and be used in more complex situations.

5.4.2 Comparison to the environment - Accordance and Trust. As in the previous section (4.4.2), after defining their personal opinion, agents might start comparing their impression to others around them, through trust formulation. However, differently from the previous case, trust can be used not only for comparison of impressions regarding the environment, but also as a mechanism for detection and even control of non-compliant behaviour. By observing neighbours, agents are able to detect anomalies in their opinions and start processes of justice restoration.

First, the accordance index metric defined in Eq. 5 is adapted for the scenario of multiple canon of justices. As an opinion is formed by different components (claims), we define $\tau_{i j}$ as the mean of each claim opinion accordance $\left(\tau_{i j}^{c}\right)$ :

$$
\tau_{i j}(t)=\frac{\sum_{c=1}^{|C|} \tau_{i j}^{c}(t)}{|C|}
$$

Where the accordance for each claim $c$ is given similarly to Eq. 5 : 


$$
\tau_{i j}^{c}(t)=1-\frac{1}{1+e^{-\kappa\left(\left|\bar{\phi}_{N_{i}}^{c}(t)-\phi_{j}^{c}(t)\right|-\epsilon_{0}^{c}\right)}}
$$

where $\bar{\phi}_{N_{i}}^{c}(t)$ is the mean opinion of claim $c$, observed in $i$ 's context:

$$
\bar{\phi}_{N_{i}}^{c}(t)=\frac{1}{|N(i)|+1} \sum_{n \in N(i) \cap\{i\}-\{j\}} \phi_{n}^{c}(t)
$$

where $N(i)$ is the set of neighbours (connections) of agent $i$.

Note that the definition of the trust threshold $\epsilon_{0}^{c}$ is crucial to the proper functioning of the algorithm. It should not be too small, otherwise agents would not be able to trust each other; it also should not be too large, otherwise non-compliant agents would be trusted and the network would not be able to detect and discourage those individuals.

Then, having calculated $\tau_{i j}(t)$, we can define the trust $\left(T_{i j}(t)\right)$ similarly to Eq. 6, that is:

$$
T_{i j}(t)= \begin{cases}0.0 & \text { if } j \notin N(j) \\ (1-\gamma) T_{i j}(t-1)+\gamma \tau_{i j}(t) & \text { if } i \neq j \wedge j \in N(j) \\ 1.0 & \text { if } i=j\end{cases}
$$

Once more, we assume that the topology of the network define the access of information of agents and limit their perception of each other.

5.4.3 Trust propagation. after the trust values are defined between pairs of agents, each agent can update its trust assessment, by exchanging impressions with their neighbours.

This process is analogous to the one described in 4.4.3, but now we propose an asynchronous process in which agents can update their trust values just with the knowledge of their neighbour's trust, instead of waiting for the update of the whole trust matrix $T$ used in Eq. 7.

This is done through a recursive process where an agent $i$ consults its neighbours $k$ about their amount of trust in a third individual $j$, connected to both $i$ and $j$. Agent $i$ then uses the trust it has on its neighbours, to update its opinion as a combination of the new opinions.

$$
T_{i j}^{\prime}(t)=\frac{\sum_{k \in N_{i j}} T_{i k}(t) T_{k j}(t)}{\sum_{k \in N_{i j}} T_{i k}(t)}
$$

where:

$$
N_{i j}=(N(i) \cap N(j)) \cup\{i\}-\{j\}
$$

Note that this process can be repeated iteratively, updating the trust until convergence. Experiments show that, in general, values stabilise with less than 50 iterations.

5.4.4 Reputation based allocation. Finally, the process of opinion and trust exchange become concrete action when, having all agents computed their opinions $\Phi_{i}(t)$ and trusts $T_{i j}$, the head can compute the order on which resources will be allocated by the agents.

This is done as follows. First, a reputation index $\left(R_{i}(t)\right)$ is computed for each agent, as the mean trust of its neighbours.

$$
R_{i}(t)=\frac{1}{\left|N_{i}\right|} \sum_{j \in N_{i}} T_{j i}(t)
$$

Then, each agent receives an urgency index $U_{i}(t)$ given by: 


$$
U_{i}(t)=R_{i}(t) *\left(1-\Phi_{i}(t)\right)
$$

The urgency index is a reliable source for judging the need of agents for resources, based on its opinion and reputation. Therefore, an allocation order is defined by sorting all agents by decreasing urgency index Following the order, the head allocates resources for agents, satisfying demands while there are resources available in the common pool.

5.4.5 Pseudocode. All the steps of the allocation algorithm are summarised in Algorithm 3.

The main strength of the presented algorithm is the fact that most of its processing is distributed among regular agents, leaving the head responsible only to sort the network's opinions, without the need of more expensive computations. This is beneficial, as it allows scalability as the algorithm is applied to large systems.

This can be clearly seen in Figure 5, where all the processing is done independently by non-head agents and there is a single step of communication to the head, responsible only for ordering the claims and returning the allocation order.

Regarding the privacy and security of the processes, the fact that all information is first computed independently by the agents and then verified by its neighbours, increases the robustness of the system against malicious manipulation - even if attempted by the head.

The algorithm also presents strong resilience against information loss. There is redundancy in the computation of personal opinions (by the agents and by its neighbours) and in the trust assessment. Therefore, even in cases where there might be communication losses, the algorithm can still function.

Finally, the process of note that this mechanism encourages two advantageous processes of self-organisation:

- If you do not ask, you do not get - each agent is encouraged to report the head their state, in order to receive resources. Thus, as communication benefits regular agents, it should happen spontaneously, without the intervention (or call) of the head;

- If you ask too much, you lose it - the reliability of an agent depends on its claims. Therefore, although there is freedom to define personal opinions that might benefit you, it can cause loss of trust on the part of its neighbours.

\subsection{Results}

In order to test algorithm 3 (DDJ), we designed experiments to evaluate the performance of the solution in diverse scenarios, including situations with non-compliant agents and in different topologies. Our investigation explores the following aspects:

- Efficiency - can DDJ efficiently distribute resources under full compliance?

- Fairness - does DDJ guarantee fair allocations in case of non-compliance?

- Robustness - what is the effect of changing network topology and behaviour?

The setting for these experiments is as follows. We consider initially a population of $n=30$ agents, connected in a ring network with neighbourhood $k=2$. Every turn, demand and availability of resources are defined randomly with $g_{i}=\operatorname{rand}(0,1)$ and $q_{i}=g_{i}+\operatorname{rand}(0,1) *\left(1-g_{i}\right)$, meaning that on average each node has only two-thirds of the resources needed.

In non-compliant rounds, the amount of resources provided is calculated by $p_{i}=g_{i} * \operatorname{rand}(0,1)$. The probability that a non-compliant will cheat in a specific turn is equal to PCheat $=0.25$. Compliant agents may also occasionally cheat, but with PCheat $=0.02$. In experiments without any non-compliant agents, we defined $P C h e a t=0.0$. Also, during every turn, each agent have its 


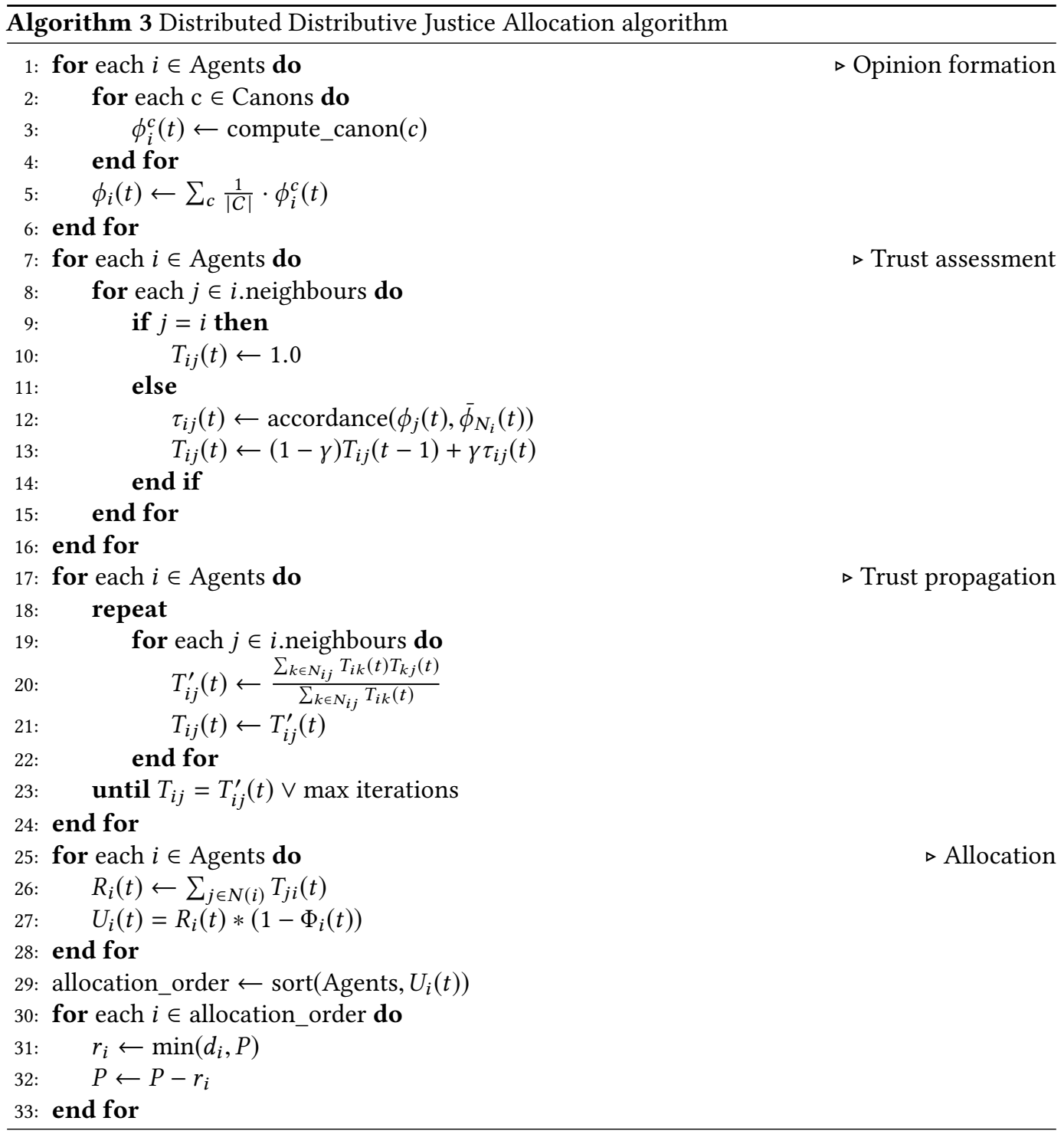

satisfaction measured by claim $\phi^{3}(t)$. If in certain turn $t$, an agent's individual satisfaction goes below a threshold $\delta=0.1$, this agent leaves the group.

For the parameters of the affinity index (Eq. 13), we defined the same coefficients $\kappa$ and $\epsilon_{0}^{c}$ for every agent. $\kappa$ and $\epsilon$ were defined experimentally (from tests of different values), assuming the values of $\kappa=15$ and $\epsilon_{0}^{c}=0.25$. Lastly, the trust reinforcement rate (Eq. 14) was defined as $\gamma=0.1$.

We did not consider equations $\phi^{6}$ and $\phi^{7}$ (canon of effort and canon of social utility) when computing personal opinions (Eq. 10 and 11). This was done because we consider only games with one active group of agents in our experiments (causing $\phi^{6}$ to be equal among all agents) and policies for head replacement were not implemented (making the value of $\phi^{7}$ insignificant). 

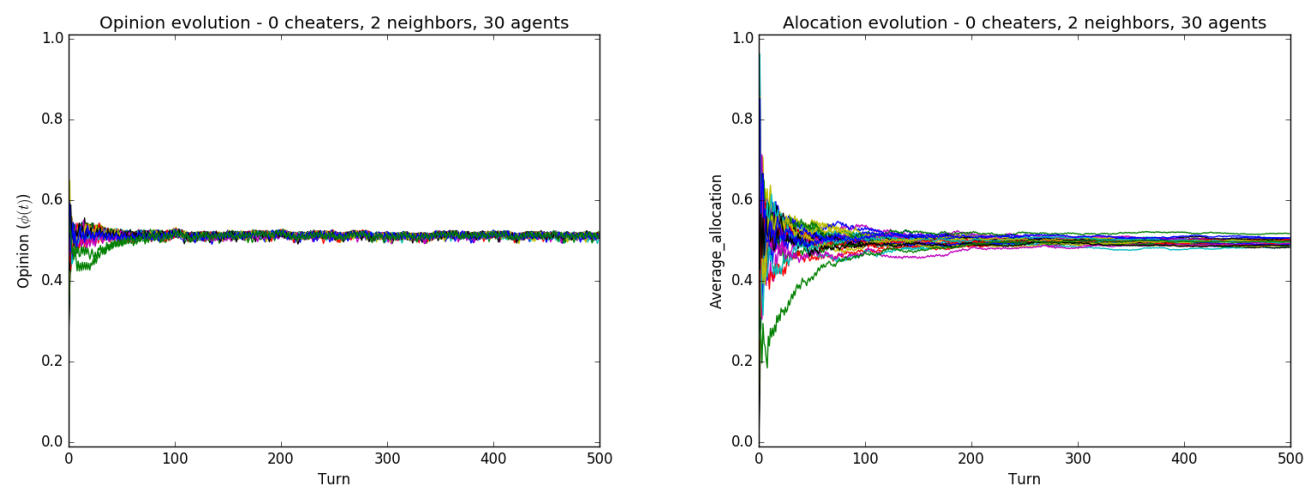

Fig. 6. Simulations of self-organising allocation. Allocation in scenario without non-compliant agents. Each coloured line represents a different agent.

5.5.1 Efficiency. The intention is that, when an agent is compliant with the game's rules, then it should be trusted and then have sufficient reputation to receive resources when it informs the head of its need for resources.

This is exactly what happens, as demonstrated by Figure 6, extracted from a game without non-compliant agents. The left image shows the opinion of each agent during 500 turns, while the right image represent agents' average allocation $\left(\frac{\sum_{t=0}^{S} r_{i}}{S}\right)$ over time.

Figure 6a shows how, in a game without cheating, the individual opinions remain in a constant and uniform value with small oscillations, indicating a general perception of fairness in the environment. Figure $6 \mathrm{~b}$ confirms this, showing that every individual receives approximately the same amount of resources over time. Thus, agents more in need are prioritised, resulting in a stable and egalitarian distribution.

5.5.2 Fairness. When there are non-compliant agents in the network, we expect that their behaviour would raise suspicion by their compliant neighbours and consequently such agents will lose reputation, so their opinions will lose strength. This will decrease the relevance of their opinions when requesting for new resources in the future.

Figure 7 presents results for a game with 10 non-compliant agents and 20 compliant. The solid lines represent non-compliant trajectories. If an agent leaves the game, its line is discontinued.

Note in Figure 7a, how the general opinion of non-compliant agents is consistently lower than of the compliant ones, justifying the mistrust. Figure $7 \mathrm{~b}$ demonstrate the network answer to this behaviour: although initially non-compliant agents might receive some resources given its dissatisfaction, after less than 100 turns, most of their reputations are so low that they stop receiving any resources, eventually leaving the game, due to dissatisfaction.

To assess quantitatively the algorithm in different scenarios, we considered its behaviour for three populations of agents submitted to the allocation: (a) het01: 20 compliant agents and 10 non-compliant; (b) hom01: 30 compliant agents with regular "dissatisfaction index" $(\beta=0.1)$ and (c) hom04: 30 compliant agents with high dissatisfaction index $(\beta=0.4)$. A high value of $\beta$ in the satisfaction index equation (third line of Table 5) represent a situation where agents can easily leave a game, if they do not receive satisfactory resources in consecutive rounds. 

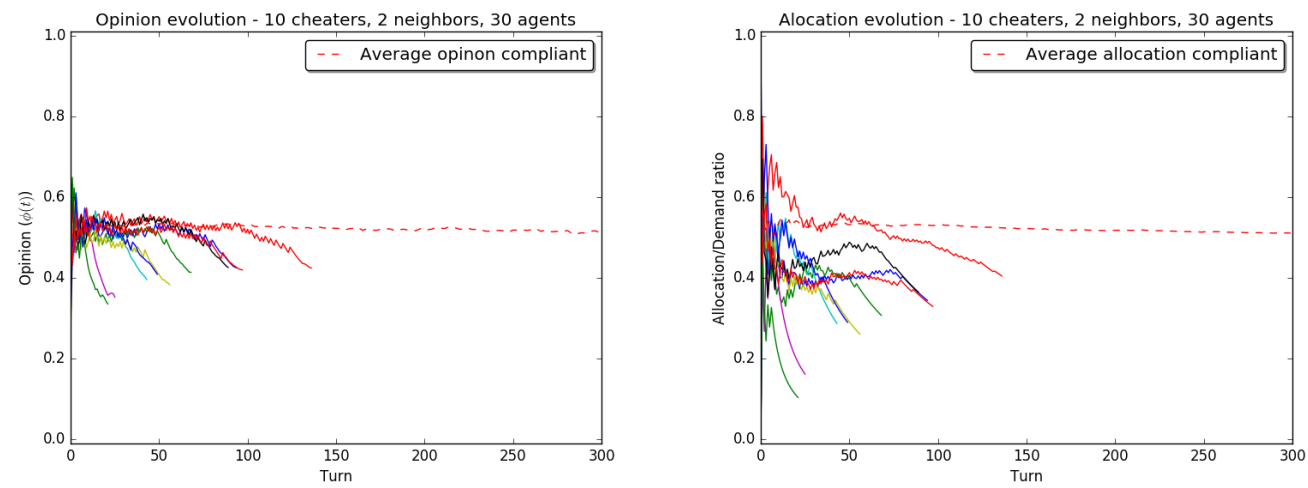

Fig. 7. Simulations of self-organising allocation, with cheating agent. Individual allocations for non-compliant agents (solid lines) compared to compliant average (dashed).

Table 6. Comparison of results for different scenarios.

\begin{tabular}{llllll}
\hline scenario & Ut.C & Ut.NC & r.C & r.NC & fairness C \\
\hline het01 & $140 \pm 7$ & $-30 \pm 4.9$ & 20 & 0 & $0.78 \pm 0.29$ \\
hom01 & $124 \pm 5$ & n/a & 30 & n/a & $0.60 \pm 0.07$ \\
hom04 & $124 \pm 5$ & n/a & 30 & n/a & $0.62 \pm 0.08$
\end{tabular}

Table 6 shows the summary of results after 50 executions of each configuration. This shows that DDJ is effective in combating non-compliant behaviour as seen in the smaller values for the non-compliant utilities $(u t . N C)$, while the compliant utilities (ut.C) remain the same. Also, it is able to correctly exclude all non-compliant agents from population het01 at the end of the execution (r.C vs $r . N C)$.

5.5.3 Robustness. The amount of connections each individual of the network has $(k)$ directly influences the amount of information it has access to and therefore the quality of judgement of non-compliant behaviour. It is surprising to see that, in the previous experiments, only two connections $(k=2)$ were enough to detect all the 10 non-compliant agents in a population of 30 agents. This might be explained by the fact that the maximum number of iterations used in the trust propagation (50) is bigger than the longest path between two agents, so even with only localised connections, agents can still indirectly receive information from even the most distant members of the network.

To explore further the network's ability to detect cheating agents, an experiment was designed where we consider a network with 100 agents and different amounts of non-compliance, by progressively increasing the number of cheaters.

Figure 8 shows, for runs with different proportions of non-compliant agents and different neighbourhood sizes $(k)$, the percentage of non-compliant agents rightly detected (i.e. the percentage of non-compliant agents that decided to leave the game due to dissatisfaction).

Note that with $k=2$ connections, the network is able to detect and punish all non-compliant agents even when they are $20 \%$ of the total population. This is remarkable, considering that for $n=100$, each agent has direct access to only $3 \%$ of the processes taking place in the network. As the number of connections increases, so does the capacity to detect non-compliant agents. When 


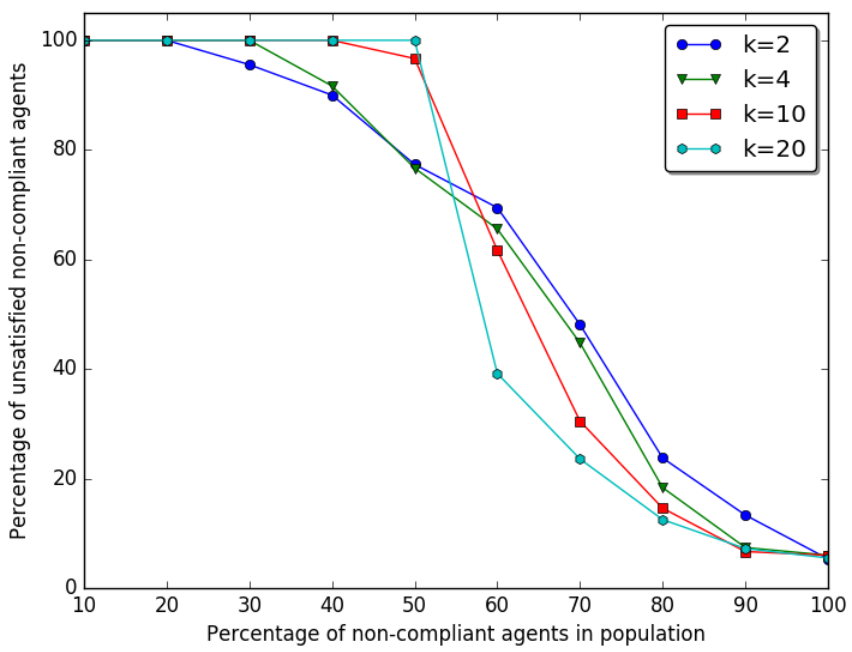

Fig. 8. Capacity of non-compliant behaviour detection, by size of neighbourhood $(k)$.

$k=20$, the network can detect all non-compliant agents, even when the population was half compliant and half non-compliant.

If the percentage of non-compliant agents is higher than $50 \%$, a different dynamic takes place, as can be seen in the right region of Figure 8. When the non-compliant population passes $50 \%$ of the total population, they start to dictate what is considered "normal" behaviour. As the trust metrics are relative to the agents' environment, in this scenario, a non-compliant behaviour will be considered more trustworthy than the behaviour of a fully compliant individual.

This creates an unsustainable environment (a tragedy of the commons) in which agents leave the game not only because they are being punished by receiving less resources, but also because the distribution of resources is inefficient as less resources are provided to the common pool. This can be seen more clearly in Figure 8 when $100 \%$ of agents are non-compliant: even with the general agreement that non-compliant behaviour is the norm (therefore no punishments are given), agents still decide to leave the game as the lack of order does not guarantee their regular access to resources.

We see, however, that in this context, networks with a higher number of connections tend to have a more sustainable environment for non-compliant agents. This can be explained by the fact that agents in those networks have more contact to different areas of the network, understanding that a non-compliant behaviour is the new 'norm' and adapting to it, instead of just trying to punish it in vain.

\subsection{Reflections and Partial Conclusions}

This section demonstrates that it is possible, using a decentralised strategy, to determine how fairly agents are being treated and to define an allocation order that prioritise agents most in need. This uses the operationalisation KM principle 7, creating a system that firstly aggregate knowledge, by considering and valuing diverse inputs (legitimate claims for resources), and secondly align knowledge, by filtering it dynamically (through trust and reputation formulations) and 
third codifying knowledge, by defining actual priority rules that govern how resource should be distributed.

Experiments show that the combination of individual opinion formation and local trust assessments can produce a scalable, efficient and reliable mechanism of justice monitoring, able to guide global decisions for which agents should be prioritised. Analytical results demonstrate the effectiveness of our algorithm in finding good allocation solutions, even when agents have limited knowledge of the processes taking place in distant parts of the network.

The results show that the way in which perceptions can be communicated and trusted by other agents is actually more important than the accurate quantification of local fairness. Therefore, we see that algorithm 3 (DDJ) is not only capable of solving direct problems of resource allocation, but also of exploring self-organising mechanisms that aggregate individual, subjective assessments, into collective, objective facts.

\section{PRINCIPLE 8: FLEXIBLE MONITORING AND SANCTIONING}

Principle 8: Processes serve as focal points/bright lines for collective action by citizens against violations and specify a hierarchy of possible punishments depending on seriousness of violation.

\subsection{Motivation}

Rules and norms generally have a very important role in regulating collectives and societies. In societies, for example, legislative systems accumulate millennia of experience on the definition of laws to organise and judge nations. However, experience also show that there is an expectation of error, so whenever there is a rule, there is also someone trying to actively break it. While we see throughout history a constant movement in the direction of organising and structuring groups, we also often perceive a strong impetus for the breaking of norms by their participants [Thoreau 1849].

Many times, this rebellious impulse can be seen as undesirable and worth to be fought against, as rule breaking may cause harm and problems. However, it is also arguable that disobedience can be a natural consequence of freedom in systems revealing an aptitude for flexibility that can be extremely beneficial.

Here, we investigate norms and sanctioning protocols in open socio-technical systems, by operationalising KM principle 8. Now, in contrast to the previous sections where the knowledge being shared was about the actual allocation and distribution of resources, this principle uses knowledge about the rule enforcement mechanisms and the agents' perception of those mechanisms.

The requirement is to build common knowledge that could contribute to successful collective action by defining how to deal appropriately with transgressions. Thus, we explore different ways to deal with non-compliance, looking particularly at the possibility of intentionally not applying sanctions directly to non-compliant behaviour. By 'appropriate' we mean taking into account contextual factors such as frequency of the offense, severity of the offense, actions of reparation, and so on. When there is a policy for sanctions, but there is choice to whether or not to apply the sanctions, we call this the Principled Violation of Policy (PVP).

The concept of PVP can be formally defined as the active and intentional decision of an agent (appointed to a role of adjudicator) of not applying a policy to which it is entitled [Axelrod 1986] More specifically, in norm-governed systems [Artikis et al. 2009] where individuals are subject to norms and sanctioning policies, PVP provides the potential to suspend or relax controlling with punishing, sanctioning and retaliation mechanisms, at the adjudicator's discretion.

We consider a situation where: (a) monitoring has costs; (b) agents are subject to accidental non-compliant behaviour; (c) norms are subject to subjective interpretation and/or (d) norms can be imposed unfairly to agents. In such scenarios, we propose the hypothesis that flexible monitoring 


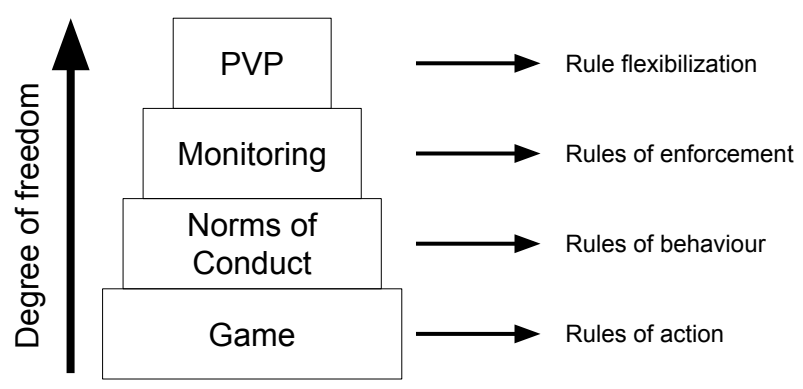

Fig. 9. Layers of society simulation used in the design of Principle 8 experiments.

and sanctioning is a more appropriate and efficient mechanism to deal with non-compliance than strict norm-enforcing strategies. Beyond that, we show how this flexibility enables the system's participants to create a more adaptable environments and offers them the potential to explore new possibilities and solutions to outstanding problems.

\subsection{Knowledge Management Principle}

A fundamental task of self-governance is to monitor, enforce and punish violation of norms [Ostrom 1990]. KM design principle 8 examines the appropriate actions to be taken in case of such violations, considering a democratic context.

Firstly agents must acknowledge the presence of pre-existing agreed processes that define the procedures upon violations, establishing clear limits and definitions (bright lines) for what constitutes a transgression and its consequences.

However, at the same time, given the plural and subjective nature of social interactions, some flexibility is desirable in the actual implementation of such procedures. Factors such as severity and context of the transgression should be taken into consideration, enabling a hierarchy of possible punishments, depending on the seriousness of the violation.

In our specific scenario, we consider that norms and predicted sanctions are in place clearly defining processes of response for non-compliance in the resource allocation process. However, flexibility in policy application enables graduated sanctioning and hierarchy of punishment, depending on the situation and context. This flexibility is guided by the Principled Violation of Policy.

\subsection{Experimental Setup}

For this set of experiments we consider a layered framework, on top of the $L P G^{\prime}$ game, as illustrated in Figure 9.

In the base layer, we use the $L P G^{\prime}$ game as the theoretical scenario where agents interact and where actions and options are defined. In this instance, we consider that the allocation is done randomly to all active players, totally unbiased.

However, building on the previous experiments, as a way to guarantee cooperation and avoid effects such as the tragedy of the commons or free-riding, a norm layer is used to enforce agents to choose certain actions and guide their behaviour, especially in situations where a rational choice of game action goes against the collective benefit.

The system's instituted norms are the following:

(1) Agents must provide its whole generation to the common pool (i.e. $p_{i}=g_{i}$ ) 


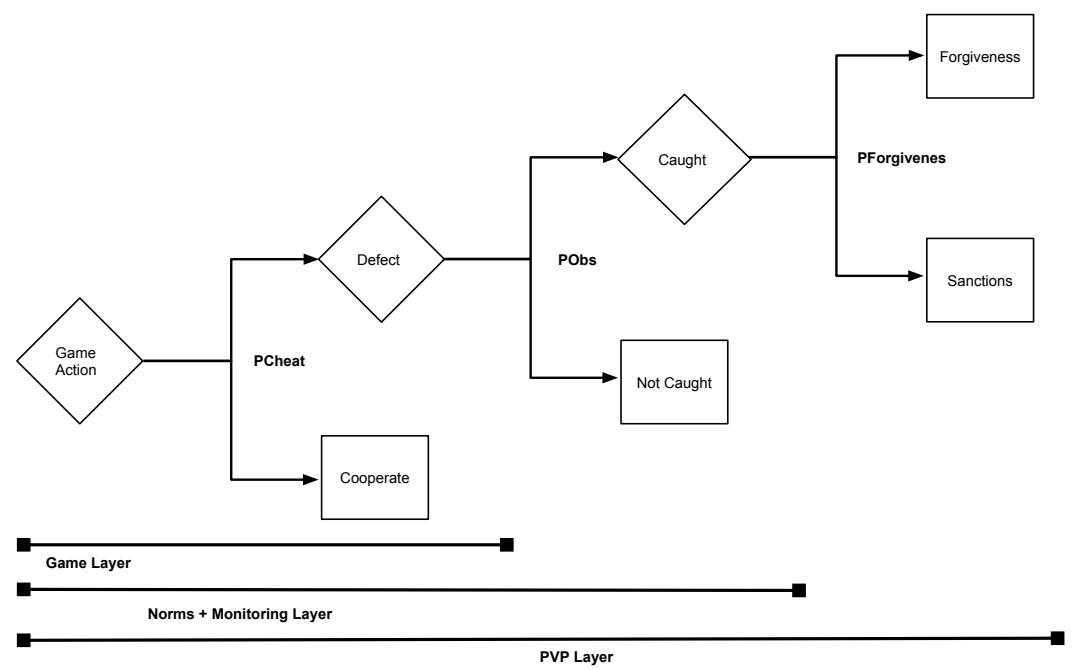

Fig. 10. Model's scheme of decisions. Based on diagram presented in [Axelrod 1986].

(2) Agents must demand only what they need $\left(d_{i}=q_{i}\right)$

(3) Agents must appropriate only what was assigned to them $\left(r_{i}^{\prime}=r_{i}\right)$

As norms often demand actions adverse to individual's objectives, there is a constant tension from an agent's perspective in the decision to comply or not comply to a rule. Therefore, a third layer is necessary, to monitor if the norms are being complied with and to determine sanctions in case of norm transgressions.

The sanctioning protocol is defined by the following enforcement rules:

- Penalty: At a given turn, if an agent violates any of the norms and a sanction is issued by an observing agent, the violator should not receive resources from the common pool for $m$ rounds;

- Mandatory Non-Repudiation: once a sanction is issued, it can not be rejected and should be applied immediately;

- Selective Non-Application: an observing agent (adjudicator) has the choice whether to apply or not a sanction, given it has observed a violation (this being the definition of PVP).

Finally, PVP would then be a fourth layer, on top of monitoring, dealing with rule flexibilization by orienting how sanctions and monitoring should be conducted and dealing with exceptions. In this layer, agents have the freedom to determine when and how sanctions should be issued and create intentional variations and reinterpretations of the enforcement rules.

\subsection{Operationalisation}

Each agent has three main parameters that rule its behaviour: PCheat, PObs and PForg, which represent the frequency of cheating, observance of neighbour's private behaviour and forgiveness respectively. Figure 10 presents a diagram showing in which layer each parameter is used in decision-making.

Cheating Behaviour. Every turn, an agent has the option to not comply with the norms and either not provide all its resources to the common pool, demand more resources than its real need, or appropriate of more resources than it was assigned. 
Although all norms have equal weight, in the context of this work we are considering only transgressions to norm \#1, cheating in the provision.

The cheating behaviour is governed by an internal variable PCheat set individually for each agent. At each turn, after having received resources, an agent makes a decision with probability $P C h e a t$ to keep part of its resources to itself, or give all its resources to the common pool (with probability $(1-$ PCheat $))$.

Monitoring and Sanctioning. A graph $G$ defines the social network that connects the $n$ agents in a game. Connected agents (neighbours) have the potential to observe each other's actions (i.e. their public and private values) and decide to investigate it further or not. If a non-compliant behaviour is identified, the observer has the option to issue a sanction or not. If at least one sanction is issued towards an agent, it should receive as punishment the impediment of receiving resources in a pre-determined number of rounds.

Therefore, each agent should define a monitoring frequency and a forgiveness policy. In our model, this is represented by $P O b s_{i}^{j}$ and $P F o r g_{i}^{j}$, that represent respectively the probability of agent $i$ observing the internal values (i.e. variables $g_{j}, p_{j}$ and $r_{j}^{\prime}$ ) of a neighbour $j$ and the probability that given a norm transgression by $j, i$ will not issue a sanction.

Note that both PObs and PForg are defined independently by each agent, implying that the monitoring and sanctioning are completely decentralised and autonomous.

$P O b s$. If we want to have realistic models, we should consider that monitoring users' behaviour comes with a cost. In our model, we transfer this cost to the agents when, upon a decision to investigate the behaviour of one of its neighbours, incur into a personal cost MonitCost, subtracted from its personal resources in the turn the observation is done.

From an agent's perspective, the rationale behind paying for the cost of investigating its neighbours is two-fold: (a) it enables the agent to know its environment and guide its future actions; (b) if non-compliance is observed, the issue of a sanction is able to restore the costs of monitoring (as the culprit won't receive resources in the next round, increasing the amount of resources available to the agent) and possibly improve the behaviour of the agent caught cheating.

PForg. Given that an agent observed a neighbour and a norm transgression is detected, the observer agent has the opportunity to issue or not a sanction towards the transgressor. This behaviour is modelled by the variable $P F$ org $_{i}^{j}$, the probability that a transgression won't be sanctioned. Different values of $P F$ org $_{i}^{j}$ imply different intensities of punishment and can be used to the development of graduated sanctions. Also, note that there is no cost associated to this action.

This flexibility is a crucial element of our model and the core mechanism that demonstrates the effects of PVP.

\subsection{Results}

Using the formal model described in the previous sections for the modelling of both the environment and agents, we can propose different experiments to investigate and discuss different scenarios that highlight the working features of flexible monitoring and sanctioning strategies.

We will particularly explore the following aspects:

- Cost-effective monitoring - how often individuals should be monitored?

- Hierarchy of punishments - what punishment strategies are more appropriate to deal with different levels of non-compliance?

- Adaptative monitoring and sanctioning - what are the advantages of being able to adapt monitoring and sanctioning policies? 
- Justice perception and policy justification - can a flexible strategy increase the awareness of justice of an institutions' members?

The simulations presented in this section considered a population of $n=30$ agents, with different topologies for $G$. The cost of monitoring was MonitCost $=0.05$ per observation and if a sanction was issued, an agent was excluded from the game for $m=10$ rounds.

After each run, we compute a utility metric per agent, as given by Equation 9, that can be aggregated and used as evidence of how well the resources were used by the agents. Moreover, a relative utility is considered by scaling a distribution of utility values between 0 and 1 .

At each turn of the $L P G^{\prime}$ game agents generate a random amount of resources between 0 and 1 $\left(g_{i}=\operatorname{rand}(0,1)\right)$ and a random demand $q_{i}=g_{i}+\operatorname{rand}(0,1) *\left(1-g_{i}\right)$. The allocation of resources is made through a random allocator which, at every round, after resources are aggregated in the common pool, orders all requests randomly and distributes it until its completion. Agents under sanction turns do not receive any resource.

6.5.1 Cost-effective Monitoring. Considering that in this system monitoring has a cost, therefore the first experiment explores the trade-off between the amount of resources spent in monitoring and its impact on general utility.

We consider a scenario where half of the agents $\left(n_{c}=15\right)$ are completely compliant $($ PCheat $=0.0)$ and are responsible for monitoring and sanctioning the other half of the agents $\left(n_{n c}\right)$, with varying levels of non-compliance. $G$ is defined as a directed bipartite graph, where every compliant agent is connected to $k=6$ non-compliant neighbours. We also consider PForg $=0.1$ for the adjudicator agents, implying that most observed norm transgressions are punished.

Considering fixed behaviours, Figure 11 shows the relative utility of the compliant group when observing different levels of fixed non-compliance (PCheat) with different intensities (PObs). Note that for all values of $P C h e a t$ (apart from $P C h e a t=0$ ) the utility initially increase as PObs grows, but finds a tipping point where the increase of $P O b s$ has little or even negative effect on it. This happens when the cost of monitoring the system becomes higher than the amount of resources recovered.

With this we show the case for a cost-effective solution. That is, complete monitoring of behaviour is not always advantageous even from a cost perspective. We know that whenever PObs $<1.0$, there is the possibility that non-compliance will occur without being detected. But if the cost of non observed transgressions is smaller than the monitoring cost, then it can be justifiable to tolerate violations in a controlled way, for the sake of the costs.

6.5.2 Hierarchy of Punishments. A sanctioning system should also determine boundaries of behaviour and an accident should be distinguished from a deliberate choice for disobedience. This is part of the graduated sanctions context, where punishments have different intensities, depending on the target. Moreover, if sanctions remove players from the game for a certain amount of rounds, punishing agents can have the undesirable effect of excluding contributions for the next rounds and decrease the overall utility.

To test the effects of graduated sanctions, we considered a scenario where a group of agents has the same fixed $P C h e a t$ (i.e. a homogeneous population) and $G$ is defined as a ring network where each agent is connected to $k=2$ neighbours. We also considered a fixed monitoring frequency, PObs $=0.5$.

Figure 12 shows utility results for different combinations of PForg and PCheat. Similarly to the results presented in 6.5.1, we can see that excessive punishment $(P F o r g=0.0)$ can be counterproductive, decreasing the general utility for lower values of PCheat. This shows that if clear 


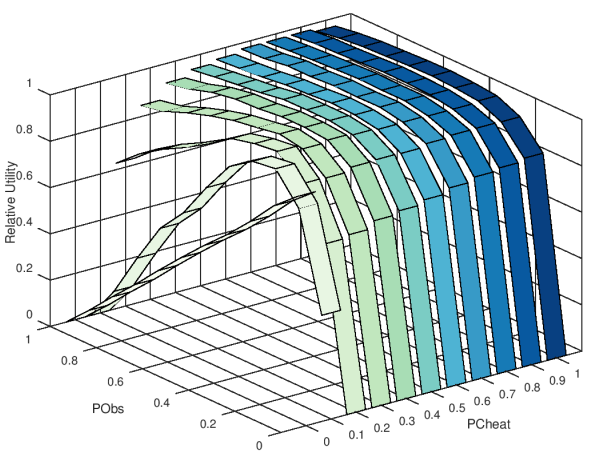

Fig. 11. Relative utility of compliant agents for different combinations of PObs and PCheat. Note that, depending on the amount of non-compliance, the increase of monitoring has small effect on the utility.

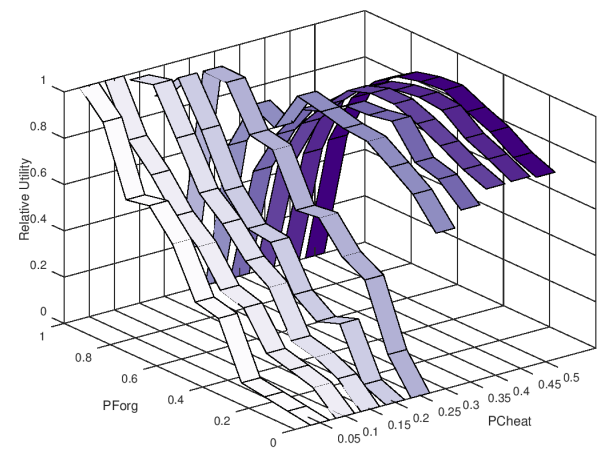

Fig. 12. Relative utility for different combinations of PForg and PCheat. Note that especially in scenarios with low non-compliance, higher utility is achieved by letting eventual non-compliant agents participate in the game, than excluding them through sanctioning.

boundaries are not defined for punishing, there is risk of backfiring, as the system unfairly punishes compliant agents.

This shows that flexible monitoring and sanctioning enables tolerance and resilience to accidents. That is, a system that tolerates certain levels of non-compliance can accommodate a bigger diversity of behaviour, without compromising utility.

6.5.3 Adaptive Monitoring and Sanctioning. The next scenario considers situations where agents do not share uniform PCheat, but present diversity of behaviour. We propose a simple reinforcement learning strategy for defining PObs and PForg, able to adapt to different scenarios.

In the adaptive strategy, agents start with pre-fixed values of $P O b s=0.5$ and $P F o r g=0.5$. PObs is increased if an agent $i$ does not receive resources in a round and decreased if, after observing the behaviour of neighbours, transgressions could not be observed. $P F o r g_{i}^{j}$ is determined independently for each neighbour $j$ and increases when $j$ is observed complying with the norms and decreases otherwise. 


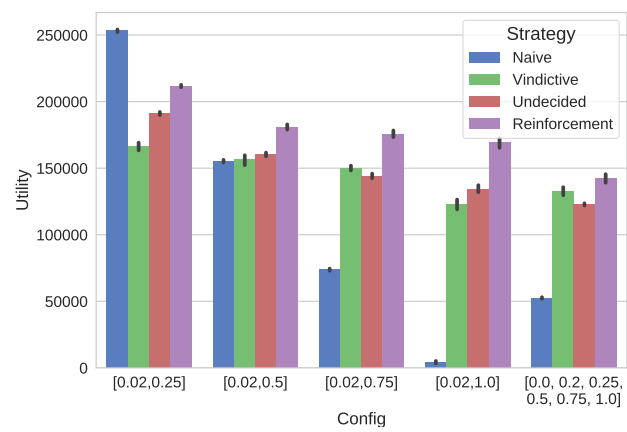

Fig. 13. Comparison of utility from different strategies, for various configurations of population. Each configuration label shows the different values of PCheat among the players' population. The reinforcement strategy is able to achieve the best results overall in different categories.

The adaptive strategy is compared to three fixed strategies, that use PObs and PForg constant:

- Naive $($ PObs $=0.0$, PForg $=1.0)-$ play the game independently of other agents, not investigating nor punishing non-compliance;

- Vindictive $(P O b s=1.0$, PForg $=0.0)$ - observe all neighbours, punish every non-compliance;

- Undecided $($ PObs $=0.5$, PForg $=0.5)$ - middle ground between Naive and Vindictive strategies.

Figure 13 shows the results for different scenarios, comparing the four strategies. Each scenario brings a population with different levels of initial non-compliance (PCheat). The labels on the horizontal axis of the figure show the different initial values of $P C h e a t$ present in the population values are distributed equally among the $n$ players of each scenario, implying the sizes of groups with the same level of non-compliance is the same in each case. As in the experiments of section 6.5.2, $G$ is a ring network, with $k=2$ neighbours. The plots show that the reinforcement approach has the best overall results considering different scenarios.

Given that fixed strategies represent games without flexible sanctioning and monitoring (the Naive condition represents systems without sanctions, while the Vindictive represents a system with complete sanctioning), we see that the presented results reveal one more feature of flexible monitoring and sanctioning: adaptability to different scenarios and behaviours. Compared to fixed policy strategies, the flexible strategy (reinforcement, in graph) is able to achieve overall better results, for different scenarios of non-compliance.

6.5.4 Justice Perception and Policy Justification. Beyond the utility results shown so far, another relevant contribution of flexible monitoring and sanctioning is the possibility of increasing agents' perception of fairness.

An experiment was conducted on which the $L P G^{\prime}$ game was played for 50000 rounds by 100 agents with diverse degrees of non-compliance (PCheat) and the reinforcement strategy was used to govern monitoring and sanctioning.

At the end of the execution each agent computed a cooperation rate - i.e. the proportion of turns in which the norms were followed $\left(p_{i}=g_{i}\right)$ in relation to the total of turns - and a punishment rate - i.e. the proportion of norm transgressions that were sanctioned in relation to the total non-compliant rounds. 


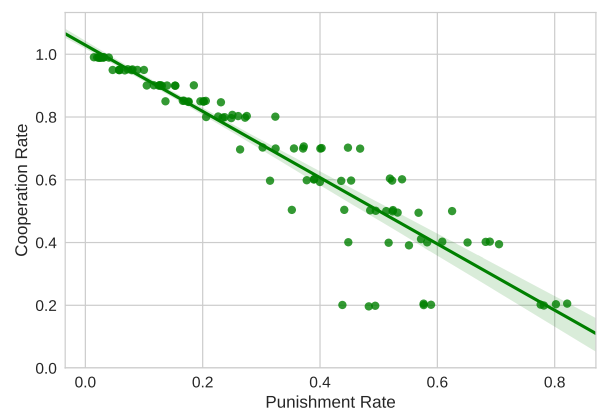

Fig. 14. Relationship between cooperation rate and punishment rate. The inverse correlation shows that agents with high levels of cooperation receive proportionally fewer sanctions than the ones who do not cooperate as often.

Figure 14 shows a scatter plot of the obtained data for all agents and a linear regression fit. By analysing the figure, we see that PVP creates an inverse correlation between agent cooperation and punishment rate, as more compliant agents receive proportionally fewer punishments.

These results indicate that a flexible strategy of monitoring and sanctioning is a mechanism for justice perception, as it enables that different behaviours receive different and fair treatments, taking into consideration agents' history.

It also shows that the differentiation in treatment can help agents to better acknowledge the reasoning behind a sanction (or a suspension of sanction), creating common knowledge on how sanctions actually work in terms of motivation and detterrent, bringing policy justification in norm-governed open systems.

\subsection{Reflections and Partial Conclusions}

This section built the case for the construction of knowledge for collective action towards norm enforcing mechanisms. We analysed alternative approaches to how norms should be enforced and proposes flexible monitoring and sanctioning policies as a pertinent and advantageous mechanism in the development of norm-governed open systems. This is achieved through what we call the Principled Violation of Policy, that is a strategy of flexibilization of monitoring and sanctioning.

The proposed formulation follows from the conceptual discussion of KM design principle 8 regarding the trade-off between having clear and predetermined processes for dealing with violations, while also maintaining subjectivity and collective participation in the application and sizing of the severity of rule enforcement.

We argue that flexibility in monitoring and sanctioning increases the potential of discovery of new solutions and creating an environment more open to diversity, adaptable and ready for reformation.

The formalisation and experiments support this claim by showing that systems with flexible monitoring and sanctioning can find solutions that are (a) cost effective; (b) hierarchical, tolerant and resilient to accidents; (c) adaptable to different scenarios and behaviour, and (d) used as a tool of justice perception and policy justification. 


\section{SUMMARY AND CONCLUSIONS}

In summary, this paper discussed the challenges in designing self-governing self-organised digital communities to address public and collective action situations. These systems need to be regulated by a type of governance based on the codification of conventional rules, which are self-organised and enacted by the system's participants themselves, and should respect some value, like fairness or sustainability.

The challenge resides in how to guarantee that rules are unrestricted enough to allow participants to adapt them to their needs, but at the same time, should be restricted to abuses of authority where a minority group manipulate it for their own benefit.

As a solution strategy, we used the results of a thorough study of classical Athenian democracy [Ober 2008] which argues that their successful governance model was due to open, transparent and inclusive strategies that were used to make information available for socially productive purposes, creating what is termed here as knowledge management processes. Athenians were able to interpret and transform raw data and unprocessed information into socially and politically valuable knowledge, by establishing sophisticated interconnected processes of knowledge aggregation, knowledge alignment and knowledge codification.

Here we argued that the same knowledge management principles can be used when approaching collective action situations in the digital era. The organisation and processing of useful knowledge in such scenarios is fundamental for the good performance of the existing processes of collective decision-making, coordination and memory. Therefore, we presented an abstraction of the studied model, supporting the strategic mechanisms discussed and translating its concepts to the digital context of socio-technical systems. Considering this new context, a set of eight design principles for democratic and sustainable self-organising socio-technical systems was formulated.

This work operationalised three of the eight design principles, demonstrating their versatility and efficiency in different experimental scenarios, in the setting of a resource allocation problem. The different experiments demonstrated how appropriate knowledge management processing can bring robust solutions to problems of accumulation of multiple perceptions of fairness; decision of resource allocation policies and finding criteria for punishment mechanisms.

First, we showed that processes of knowledge aggregation and knowledge alignment modelled as opinion formation, trust and influence, are able to value, but also filter agent's epistemic diversity. Thus, it is possible to access reliable feedback knowledge of a system status, by representing and satisfying shared values, identifying experts and locus of expertise, while rejecting opportunistic and misleading information.

Second, we extended the knowledge management processes towards executive action, creating a structure of communication, trust and reputation enabling systems to administer power fairly by defining rules for resource allocation and distribution. This was done by the codification of aligned knowledge and the creation of clear procedural rules that guarantee rightful and accurate knowledge for decision taking, even in the context of devious and non-compliant behaviour.

Third, we investigated how knowledge management processes can be used not only for the decision on how to handle resources and communicate agents perceptions of fairness, but also for promoting more complex and comprehensive rule enforcement mechanisms. We discussed monitoring and sanctioning processes, realising that flexibility and forgiveness can be used to generate hierarchical behaviour, but also tolerance. This scenario is crucial to the perception of fairness and also to enable learning from the agents.

Thus, the three operationalisations provide an empirical demonstration, through formal models and experimental results, that the same Knowledge Management Principles experienced thousands 
of year ago in Ancient Greece can still be successfully applied to modern socio-technical systems, typical of the digital age.

Although currently just few of all the identified design principles were implemented, we believe that their results already present motivating evidence of its suitability and appropriateness in the context being considered. Therefore, implementation of the remaining principles are planned for future work.

Moreover, the prospects of the current experiments lead us to argue, in conclusion, that this approach to the design of socio-technical systems can provide a balance between restricted and un-restricted self-modification of conventional rules, and can thus provide the foundations for sustainable and democratic self-governance in socio-technical systems.

\section{ACKNOWLEDGMENTS}

The first author is supported by the National Council for Scientific and Technological Development (CNPq), Brazil. We also would like to thank the helpful and pertinent comments of anonymous reviewers in previous versions of this work that contributed to it improvement.

This work is an extension of works presented previously at the context of IEEE International Conference on Self-Adaptive and Self-Organizing Systems (SASO) and IEEE International Workshops on Foundations and Applications of Self* Systems (FAS*W).

The Knowledge Management Processes framework and the design principles were first introduced in [Pitt et al. 2017]; Principle 6 (Voices of Justice) in [Kurka and Pitt 2016b]; Principle 7 (Distributed Distributive Justice) in [Kurka and Pitt 2016a]; and Principle 8 (Flexible Monitoring and Sanctioning) in [Kurka and Pitt 2017].

\section{REFERENCES}

Eduardo Araral. 2014. Ostrom, Hardin and the Commons: A Critical Appreciation and a Revisionist View. Environmental Science \& Policy 36 (Feb. 2014), 11-23. https://doi.org/10.1016/j.envsci.2013.07.011

Joe Armstrong, Robert Virding, Claes Wikström, and Mike Williams. 1993. Concurrent Programming in ERLANG. (1993).

Alexander Artikis. 2012. Dynamic Specification of Open Agent Systems. Journal of Logic and Computation 22, 6 (Dec. 2012 ), 1301-1334. https://doi.org/10.1093/logcom/exr018

Alexander Artikis, Marek Sergot, and Jeremy Pitt. 2009. Specifying Norm-Governed Computational Societies. ACM Trans. Comput. Logic 10, 1 (Jan. 2009), 1:1-1:42. https://doi.org/10.1145/1459010.1459011

Robert Axelrod. 1986. An Evolutionary Approach to Norms. American Political Science Review 80, 4 (Dec. 1986), $1095-1111$. https://doi.org/10.1017/S0003055400185016

Geoffrey Brennan and Philip Pettit. 2004. The Economy of Esteem: An Essay on Civil and Political Society. OUP Oxford.

John Seely Brown and Paul Duguid. 2000. The Social Life of Information. Harvard Educational Review 71, 1 (2000), $151-152$.

Jeffrey A. Burke, Deborah Estrin, Mark Hansen, Andrew Parker, Nithya Ramanathan, Sasank Reddy, and Mani B. Srivastava. 2006. Participatory Sensing. In International Workshop on World-Sensor-Web. 117-134.

Ruth Berins Collier and David Collier. 1991. Shaping the Political Arena. Princeton University Press, Princeton.

Sue E. S. Crawford and Elinor Ostrom. 1995. A Grammar of Institutions. American Political Science Review 89, 3 (Sept. 1995), 582-600. https://doi.org/10.2307/2082975

Marcello D’Agostino and Corrado Sinigaglia. 2010. Epistemic Accuracy and Subjective Probability. In EPSA Epistemology and Methodology of Science. Springer, 95-105.

Thomas H. Davenport and Laurence Prusak. 1998. Working Knowledge: How Organizations Manage What They Know. Harvard Business Press.

Morris H. DeGroot. 1974. Reaching a Consensus. F. Amer. Statist. Assoc. 69, 345 (1974), 118-121.

Nancy M. Dixon. 2000. Common Knowledge: How Companies Thrive by Sharing What They Know. Harvard Business School Press, Boston, Mass.

Paul Erdös and Alfréd Rényi. 1959. On Random Graphs. Publicationes Mathematicae Debrecen 6 (1959), $290-297$.

Rino Falcone and Cristiano Castelfranchi. 2001a. Social Trust: A Cognitive Approach. In Trust and Deception in Virtual Societies. Springer, 55-90.

Rino Falcone and Cristiano Castelfranchi. 2001b. The Socio-Cognitive Dynamics of Trust: Does Trust Create Trust? In Trust in Cyber-Societies. Springer, 55-72. 
Batya Friedman, Peter H. Kahn, Alan Borning, and Alina Huldtgren. 2013. Value Sensitive Design and Information Systems. In Early Engagement and New Technologies: Opening up the Laboratory. Springer, Dordrecht, 55-95. https: //doi.org/10.1007/978-94-007-7844-3_4

Simon Gächter. 2006. Conditional Cooperation: Behavioral Regularities from the Lab and the Field and Their Policy Implications. School of Economics, University of Nottingham, Discussion Papers 2006-03 (2006).

Corrado Gini. 1912. Variabilità e Mutabilità. Reprinted in Memorie di metodologica statistica (Ed. Pizetti E, Salvemini, T). Rome: Libreria Eredi Virgilio Veschi (1912).

Russell Hardin. 2013. The Free Rider Problem. In The Stanford Encyclopedia of Philosophy (spring 2013 ed.), Edward N. Zalta (Ed.). Metaphysics Research Lab, Stanford University.

F. A. Hayek. 1945. The Use of Knowledge in Society. The American Economic Review 35, 4 (1945), 519-530.

Charlotte Hess and Elinor Ostrom. 2007. Understanding Knowledge as a Commons. The MIT Press.

David Burth Kurka and Jeremy Pitt. 2016a. Distributed Distributive Justice. In 2016 IEEE 10th International Conference on Self-Adaptive and Self-Organizing Systems (SASO). 80-89. https://doi.org/10.1109/SASO.2016.14

David Burth Kurka and Jeremy Pitt. 2016b. Voices of Justice: Finding Consensus in the Multitude of Claims. In 2016 IEEE 1st International Workshops on Foundations and Applications of Self* Systems (FAS*W). 174-179. https://doi.org/10.1109/FASW.2016.47

David Burth Kurka and Jeremy Pitt. 2017. The Principled Violation of Policy: Norm Flexibilization in Open Self-Organising Systems. In 2017 IEEE 2nd International Workshops on Foundations and Applications of Self* Systems (FAS $\left.{ }^{*} W\right)$. Tucson, Arizona, USA, 33-38. https://doi.org/10.1109/FAS-W.2017.117

Richard A. Lanham. 2006. The Economics of Attention: Style and Substance in the Age of Information. University of Chicago Press.

Lawrence Lessig. 2009. Code: And Other Laws of Cyberspace. ReadHowYouWant. com.

P. R. Lewis, L. Esterle, A. Chandra, B. Rinner, and X. Yao. 2013. Learning to Be Different: Heterogeneity and Efficiency in Distributed Smart Camera Networks. In 2013 IEEE 7th International Conference on Self-Adaptive and Self-Organizing Systems. 209-218. https://doi.org/10.1109/SASO.2013.20

Sam Macbeth and Jeremy Pitt. 2015. Self-Organising Management of User-Generated Data and Knowledge. The Knowledge Engineering Review 30, 3 (May 2015), 237-264. https://doi.org/10.1017/S026988891400023X

Robert Michels. 1915. Political Parties: A Sociological Study of the Oligarchical Tendencies of Modern Democracy. Hearst's International Library Company.

Josiah Ober. 2008. Democracy and Knowledge. Princeton University Press.

Josiah Ober. 2013. Democracy's Wisdom: An Aristotelian Middle Way for Collective Judgment. American Political Science Review 107, 1 (Feb. 2013), 104-122. https://doi.org/10.1017/S0003055412000627

Elinor Ostrom. 1990. Governing the Commons: The Evolution of Institutions for Collective Action. Cambridge University Press. Cambridge University Press.

Scott E. Page. 2007. The Difference: How the Power of Diversity Creates Better Groups, Firms, Schools, and Societies. Vol. 6. Princeton University Press.

Philip Pettit and Geoffrey Brennan. 2004. The Economy of Esteem. Oxford: Oxford University Press.

Rosalind Wright Picard. 1997. Affective Computing. MIT Press, Cambridge.

Jeremy Pitt, Dídac Busquets, and Sam Macbeth. 2014. Distributive Justice for Self-Organised Common-Pool Resource Management. ACM Transactions on Autonomous and Adaptive Systems 9, 3 (Oct. 2014), 1-39. https://doi.org/10.1145/ 2629567

Jeremy Pitt, Dídac Busquets, and Régis Riveret. 2015. The Pursuit of Computational Justice in Open Systems. AI \& SOCIETY 30, 3 (Aug. 2015), 359-378. https://doi.org/10.1007/s00146-013-0531-6

Jeremy Pitt, Josiah Ober, and Ada Diaconescu. 2017. Knowledge Management Processes and Design Principles for SelfGoverning Socio-Technical Systems. In 2017 IEEE 2nd International Workshops on Foundations and Applications of Self* Systems $\left(F A S^{*} W\right) .97-102$. https://doi.org/10.1109/FAS-W.2017.127

Jeremy Pitt and Julia Schaumeier. 2012. Provision and Appropriation of Common-Pool Resources without Full Disclosure. In PRIMA 2012: Principles and Practice of Multi-Agent Systems. Springer, Berlin, Heidelberg, 199-213. https://doi.org/10. 1007/978-3-642-32729-2_14

Jeremy Pitt, Julia Schaumeier, and Alexander Artikis. 2012. Axiomatization of Socio-Economic Principles for Self-Organizing Institutions. ACM Transactions on Autonomous and Adaptive Systems 7, 4 (Dec. 2012), 1-39. https://doi.org/10.1145/ 2382570.2382575

Nicholas Rescher. 1966. Distributive fustice: A Constructive Critique of the Utilitarian Theory of Distribution. Irvington Publishers.

Henry M. Robert and Sarah Corbin Robert. 2011. Robert's Rules of Order Newly Revised (11th ed., a new and enlarged ed. / by sarah corbin robert ... [et al.] ed.). Da Capo Press, Philadelphia, PA. 
Agnieszka Rychwalska and Magdalena Roszczynska-Kurasinska. 2017. Value Sensitive Design for Peer Production Systems Mediating Social Interactions. IEEE Technology and Society Magazine 36, 3 (Sept. 2017), 48-55. https://doi.org/10.1109/ MTS.2017.2728737

Jan-Philipp Steghöfer, Gerrit Anders, Florian Siefert, and Wolfgang Reif. 2013. A System of Systems Approach to the Evolutionary Transformation of Power Management Systems. In Informatik 2013, 43. Jahrestagung Der Gesellschaft Für Informatik e.V. (GI), Informatik Angepasst an Mensch, Organisation Und Umwelt, 16.-20. September 2013, Koblenz, Deutschland (LNI), Matthias Horbach (Ed.), Vol. 220. GI, 1500-1515.

Peter Suber. 1990. The Paradox of Self-Amendment: A Study of Law, Logic, Omnipotence, and Change. Peter Lang International Academic Publishers.

Henry David Thoreau. 1849. The Resistance to Civil Government. In Aesthetic Papers. Boston and New York: The Editor and G.P. Putnam, 189-211.

Duncan James Watts and Steven Henry Strogatz. 1998. Collective Dynamics of 'small-World' Networks. Nature 393, 6684 (June 1998), 440-2. https://doi.org/10.1038/30918 